Interfaces and Free Boundaries 14 (2012), 231-272

DOI $10.4171 / \mathrm{IFB} / 281$

\title{
Finite element methods for director fields on flexible surfaces
}

\author{
SÖREN BARTELS \\ Universität Bonn, Institut für Numerische Simulation, Wegelerstr. 6, 53115 Bonn, Germany \\ E-mail: bartels@ins.uni-bonn.de \\ GEORG DOLZMANN \\ Fakultät für Mathematik, Universität Regensburg, 93040 Regensburg, Germany \\ RICARDO H. NOCHETTO \\ Mathematics Department and Institute for Physical Science and Technology, University of \\ Maryland, College Park, MD 20742, U.S.A. \\ ALEXANDER RAISCH \\ Universität Bonn, Institut für Numerische Simulation, Wegelerstr. 6, 53115 Bonn, Germany \\ [Received 14 June 2011 and in revised form 16 March 2012]

\begin{abstract}
We introduce a nonlinear model for the evolution of biomembranes driven by the $L^{2}$-gradient flow of a novel elasticity functional describing the interaction of a director field on a membrane with its curvature. In the linearized setting of a graph we present a practical finite element method (FEM), and prove a priori estimates. We derive the relaxation dynamics for the nonlinear model on closed surfaces and introduce a parametric FEM. We present numerical experiments for both linear and nonlinear models, which agree well with the expected behavior in simple situations and allow predictions beyond theory.
\end{abstract}

2010 Mathematics Subject Classification: Primary 35K55, 74K15.

Keywords: Finite elements, surfaces, biomembranes, surfactants, director fields

\section{Introduction and discussion of the proposed model}

The question of how to predict the shape of a cell bounded by a lipid bilayer membrane has inspired a significant body of research in the past twenty years ranging from purely mechanical descriptions to advanced mathematical analysis. We refer, e.g., to the papers [10, 19, 21, 22] for the discussion of the shape of a red blood cell and the basic models developed for this purpose. Excellent reviews of the topic can be found in [27, 30]. Almost all of these models share the basic structure given by an energy functional $E(\Gamma)$ depending on the shape of the cell which is identified with a surface $\Gamma$,

$$
E(\Gamma)=\int_{\Gamma}\left(\frac{\kappa_{0}}{2}\left(H-H_{0}\right)^{2}+\frac{\kappa_{G}}{2} K\right) \mathrm{d} \sigma .
$$

Here $H$ is the mean curvature and $K$ the Gauss curvature of $\Gamma$ and $\kappa_{0}$ and $\kappa_{G}$ are the associated moduli of elasticity. By Gauss-Bonnet, the integral of $K$ is a topological constant on a closed surface and can be neglected for evolutions in one topological class of surfaces. The quantity $H_{0}$ is usually referred to as spontaneous curvature and describes the preferred value of curvature induced by the ambient space on a membrane in equilibrium. Such $H_{0}$ might be constant, the usual choice, but 
it might also depend on another variable such as the bilipid concentration [7, 13, 17, 18, 28, 33]. Alternatively, $H_{0}$ might be induced by an underlying director field as in [26] and our models below. The first variation of (1.1) with respect to $\Gamma$ is given in [4] for $H_{0}$ constant and in [12] for $H_{0}$ depending on position.

In a broader context, the question to find the shape of a cell is surprisingly similar to the related problem of determining the shape of an interface between two immiscible liquids with or without surfactants. The prediction of the structure and the elastic properties of such interfaces is still a challenging problem in applied mathematics and physics and has been investigated by a wide range of techniques reaching from molecular dynamics simulations to continuum descriptions in coarse grained models; see [26] that inspired this work. These similarities motivate us to explore model energies for membranes which combine classical elasticity terms like those present in (1.1) with terms which couple the local orientation of the surfactants or the lipid molecules with the curvature of the interface or the membrane, respectively; we started this investigation in [6]. These energy contributions are relevant in the gel phase of the membrane.

In this paper we investigate a novel model for the shape of a lipid bilayer membrane which takes into account a coupling between the curvature $H=-\operatorname{div}_{\Gamma} v$ of the membrane $\Gamma$ and the local orientation of the lipid molecules, described by a director field $n$. The nonlinear model is governed by the energy

$$
E(\Gamma, n)=\int_{\Gamma}\left(\frac{1}{2}\left|\operatorname{div}_{\Gamma} v-\delta \operatorname{div}_{\Gamma} n\right|^{2}+\frac{\lambda}{2}\left|\nabla_{\Gamma} n\right|^{2}\right) \mathrm{d} \sigma
$$

where $\operatorname{div}_{\Gamma}$ and $\nabla_{\Gamma}$ are the tangential divergence and gradient operators and $\lambda>0$. Comparing with (1.1), we can interpret $H_{0}=-\delta \operatorname{div}_{\Gamma} n$ with $\delta \in \mathbb{R}$ as an induced spontaneous curvature on $\Gamma$ due to the coupling with $n$. In order to develop an effective approximation scheme, we first linearize this model locally in a flat region of $\Gamma$ and represent $\Gamma$ as a graph with height $u$ (Monge gauge). The resulting model is a special case of that introduced by Laradji and Mouritsen [26]. We study this model, propose a practical FEM for an $L^{2}$-gradient flow of the linearized functional, and show a priori estimates, which lead to existence of a limiting solution pair $(u, n)$. We also explore the dynamics of defects using our FEM. We next return to the nonlinear functional (1.2), derive an $L^{2}$-gradient flow, propose a practical FEM for its solution, and show simulations of defects. The insight gathered from the linearized graph case turns out to be useful in understanding the nonlinear regime.

\subsection{A model for surfactants}

The starting point of our analysis is the Ginzburg-Landau model in Laradji and Mouritsen [26] which was originally developed for surfactant monolayers at liquid-liquid interfaces with a locally varying density of surfactants $\phi$. The formulation assumes that this interface is given by a twodimensional surface $\Gamma$ in the three-dimensional ambient space described by a height function $u$ : $\Omega \rightarrow \mathbb{R}$. The model in [26], which is discussed below, is an attempt to match deviations from the bending energy model (1.1) with $H_{0}=0$ for low wave numbers, which were detected via molecular dynamics computations. The total energy of a configuration is assumed to be given by 
(see Appendix A in [26])

$$
\begin{aligned}
\mathcal{F}(u, \phi, n)=\int_{\Omega}\left(\xi \sqrt{1+|\nabla u|^{2}}+\frac{\kappa}{2}|\operatorname{div} v|^{2}+\frac{a}{2} \phi^{2}+\frac{c}{2}|\nabla \phi|^{2}-\mu_{s} \phi\right. \\
\left.+\frac{g(\phi)}{2}|n|^{2}-h(\phi) v \cdot n+\frac{k(\phi)}{2}|\operatorname{div} n|^{2}-\frac{\ell(\phi)}{2} \operatorname{div} v \operatorname{div} n\right) \mathrm{d} x
\end{aligned}
$$

with suitable constants $\xi, \kappa, a, c, \mu_{s}$ and nonnegative functions $\phi, h, g, k$, and $\ell$. Here $\nabla$ and div denote the planar differential operators gradient, i.e., $\nabla z=\left(\partial_{1} z, \partial_{2} z\right)$ for a scalar function $z$, and divergence, i.e., $\operatorname{div} F=\partial_{1} F_{1}+\partial_{2} F_{2}$ for a vectorfield $F=\left(F_{1}, F_{2}, F_{3}\right)$, whereas $v=$ $(-\nabla u, 1) / \sqrt{1+|\nabla u|^{2}}$ is the normal to the graph of $u$. In [26] it is shown that the surface tension $\xi$ is vanishingly small for densities $\phi$ close to one. Therefore we may assume that $\xi \approx 0$ and that $\phi$ is nearly equal to 1 and discard all terms depending on $\phi$ and $\xi$ in $\mathcal{F}$. As a further simplification and in order to focus on the interaction of orientation and curvature, we assume that $n$ is a unit vector and we omit for the moment the term proportional to $v \cdot n$ which favors alignment of $n$ along $v$. This leads to the following model which contains the essential features

$$
\mathcal{F}(u, n)=\int_{\Omega}\left(\frac{\kappa}{2}|\operatorname{div} v|^{2}+\frac{k}{2}|\operatorname{div} n|^{2}-\frac{\ell}{2} \operatorname{div} v \operatorname{div} n\right) \mathrm{d} x,
$$

with constant parameters $\kappa, k, \ell$. Upon completing the square, one obtains

$$
\mathcal{F}(u, n)=\int_{\Omega}\left(\frac{\kappa}{2}\left(\operatorname{div} v-\frac{\ell}{2 \kappa} \operatorname{div} n\right)^{2}+\left(\frac{k}{2}-\frac{\ell^{2}}{8 \kappa}\right)|\operatorname{div} n|^{2}\right) \mathrm{d} x .
$$

Comparing with (1.1) and (1.2), we interpret this formula as saying that the local arrangement of the surfactants leads to a (position dependent) spontaneous curvature

$$
H_{0}=-\frac{\ell}{2 \kappa} \operatorname{div} n
$$

which becomes less important for large values of the bending rigidity $\kappa$. We finally observe that in order to bound the energy it is sufficient to assume that

$$
\frac{k}{2}-\frac{\ell^{2}}{8 \kappa} \geqslant 0 \text {. }
$$

The corresponding positive convex term $|\operatorname{div} n|^{2}$ in the energy gives us coercivity of the functional $\mathcal{F}(u, n)$ but it is insufficient for devising a practical numerical scheme, deriving a priori bounds for discrete solutions which allow passing to the limit, and showing existence of a minimizing pair $(u, n)$. We thus modify the model upon replacing $|\operatorname{div} n|^{2}$ with the usual Frank energy $|\nabla n|^{2}$ of the director field $n$, which is ubiquitous in the theory of liquid crystals. As the maximal mesh size tends to zero we may pass to the limit for $n$, in view of the enhanced $H^{1}$ regularity, as well as to enforce the unit length constraint on $n$ via a projection method due to Alouges [1], and extended in [5] to FEM. Such a projection does not increase the energy of the Dirichlet integral, but the analogous assertion is not true for the energy of the divergence. 


\subsection{A model for biomembranes}

Our interest in augmented Canham-Helfrich models originates in the search for models that allow one to predict the experimentally observed coarsening mechanisms in membranes in the gel phase based on recombination of topological defects [23]. Related models, based on the assumption that this recombination is driven by an interaction between the director field and the curvature, have been proposed in [32] and analyzed in [6]. See also [20] for a closely related approach.

In the model in [32] the lipid monolayer is considered in the gel phase and it is assumed that the director field is oriented in a fixed angle relative to the surface normal [29]. Therefore it suffices to study the tangential part $m$ of the director field which is itself a vector field of fixed length. The related energy functional in a linearized setting is

$$
E(u, m)=\frac{\kappa}{2} \int_{\Omega}|\Delta u|^{2} \mathrm{~d} x+\frac{C_{q}}{2} \int_{\Omega}|\nabla m|^{2} \mathrm{~d} x-\delta \int_{\Omega} D^{2} u:\left(m \otimes m-\frac{1}{2} I\right) \mathrm{d} x,
$$

subject to a length constraint for $m$. Our numerical experiments for a rigidly imposed length constraint show that the coupling between $u$ and $m$ is too weak in the regime of parameters which define a well-posed minimization problem in order to simulate the observed recombination of defects [6]. The coupling proposed in the present model is stronger in the sense that it involves one more derivative. It also allows a direct extension to the nonlinear model (1.2) on closed surfaces; cf. Section 1.6.

\subsection{Linear model on graphs}

We give a linearized version of (1.2) for surfactants and augment the obtained energy by a term which penalizes deviations of the out of plane component from a given value to model biomembranes. We focus on the local situation in which the surface $\Gamma$ is described by the graph of a function $u: \Omega \rightarrow \mathbb{R}^{3}$ with $\Omega \subset \mathbb{R}^{2}$ convex. Moreover, we assume that the displacements are small,

$$
|\nabla u| \ll 1 .
$$

This yields $\sqrt{1+|\nabla u|^{2}} \approx 1$ as well as $v \approx(-\nabla u, 1)$, whence $\operatorname{div}_{\Gamma} v \approx-\Delta u$. Moreover, we have

$$
\nabla_{\Gamma} n \approx \nabla n, \quad \operatorname{div}_{\Gamma} n \approx \operatorname{div} n_{p},
$$

where $n_{p}$ stands for the tangential part of the director field $n=\left(n_{1}, n_{2}, n_{3}\right)$, that is, $n_{p}=\left(n_{1}, n_{2}\right)$, and $\nabla$, div are the planar differential operators. We are now ready to write the linearized energy: find $u \in H^{2}(\Omega)$ with $u=u_{D}$ on $\partial \Omega, u_{D} \in H^{2}(\Omega), n \in H^{1}\left(\Omega ; \mathbb{R}^{3}\right)$ with $n=n_{D}$ on $\partial \Omega$, $n_{D} \in H^{1}\left(\Omega ; \mathbb{S}^{2}\right)$ and $\mu \in L^{1}(\Omega)$ as stationary points of the integral

$$
\begin{aligned}
E(u, n, \mu)=\frac{1}{2} \int_{\Omega} \mid \Delta u+\delta & \left.\operatorname{div} n_{p}\right|^{2} \mathrm{~d} x \\
& +\frac{1}{2} \int_{\Omega}|\nabla n|^{2} \mathrm{~d} x+\frac{1}{2} \int_{\Omega} \mu\left(|n|^{2}-1\right) \mathrm{d} x-\int_{\partial \Omega} g \partial_{\nu} u \mathrm{~d} S .
\end{aligned}
$$

Note that $\mu \in L^{1}(\Omega)$ is the Lagrange multiplier for the nonlinear constraint $n \in \mathbb{S}^{2}$ and that $g$ is related to the boundary values for the mixed method we discuss below. This model captures the essential features of the simplified linear model of Section 1.1 with energy $\mathcal{F}(u, n)$. 
To model surfactants we do not impose an angle between $v$ and $n$, which typically tend to align in the gel phase of the membrane. To model biomembranes, instead, we penalize the deviation of $v \cdot n \approx n_{3}$ from a prescribed value $\xi_{0}$ via

$$
\frac{1}{2 \varepsilon^{2}} \int_{\Omega}\left(\left|n_{3}\right|^{2}-\xi_{0}^{2}\right)^{2}
$$

with small parameter $\varepsilon>0$. This term being lower order does not cause difficulties in the numerical method or the passage to the limit and will thus be ignored for the subsequent discussion until Section 4.

\subsection{Relaxation dynamics for surfactants}

To detect critical points we suggest a relaxation dynamics given by an $L^{2}$-gradient flow, i.e., we assume that there exist constants $\Gamma_{u}$ and $\Gamma_{n}>0$ such that

$$
\begin{array}{ll}
\left\langle\partial_{t} u, v\right\rangle=-\Gamma_{u}\left\langle\frac{\delta E}{\delta u}, v\right\rangle \quad \text { for all } v \in H^{2}(\Omega) \cap H_{0}^{1}(\Omega), \\
\left\langle\partial_{t} n, m\right\rangle=-\Gamma_{n}\left\langle\frac{\delta E}{\delta n}, m\right\rangle \quad \text { for all } m \in H_{0}^{1}\left(\Omega, \mathbb{R}^{3}\right) .
\end{array}
$$

For simplicity we assume in the following that the units are chosen in such a way that $\Gamma_{u}=\Gamma_{n}=1$. If we include the equilibrium condition for the Lagrange multiplier in our equations, then we obtain the following coupled system of partial differential equations: for all $v \in H^{2}(\Omega) \cap H_{0}^{1}(\Omega)$, for all $m \in H_{0}^{1}\left(\Omega ; \mathbb{R}^{3}\right)$, and for all $\eta \in L^{1}(\Omega)$,

$$
\begin{aligned}
\left\langle\partial_{t} u, v\right\rangle & =-\left(\Delta u+\delta \operatorname{div} n_{p}, \Delta v\right)+\int_{\partial \Omega} g \partial_{\nu} v \mathrm{~d} S, \\
\left\langle\partial_{t} n, m\right\rangle & =-\left(\Delta u+\delta \operatorname{div} n_{p}, \delta \operatorname{div} m_{p}\right)-(\nabla n, \nabla m)-(\mu n, m), \\
0 & =\frac{1}{2}\left(\eta,|n|^{2}-1\right) .
\end{aligned}
$$

Hereafter we write for simplicity $(\cdot, \cdot)$ for the inner product in $L^{2}$. We impose the following boundary conditions provided by the setting of the model,

$$
u=u_{D}, n=n_{D} \text { on } \partial \Omega,
$$

and we need to choose a second boundary condition for the fourth order equation involving $u$. Such a condition is implicit in the equation for $u_{t}$ above because integration by parts gives formally $\tilde{z}=g$ with

$$
\tilde{z}=\Delta u+\delta \operatorname{div} n_{p} .
$$

Despite the fact that this quantity is a priori only in $L^{2}$, we prove that there exists a solution with $\tilde{z} \in H^{1}$ so that the boundary condition $\tilde{z}=g$ is well-posed. Note that this is a natural condition at first sight in the energy minimization but it becomes essential for the operator splitting: we use a mixed method for the variables $u$ and $z=\tilde{z}-g$ with homogeneous Dirichlet boundary conditions. 
Finally we collect the equations in their strong form:

$$
\begin{aligned}
\tilde{z} & =\Delta u+\delta \operatorname{div} n_{p}, & & \left.\tilde{z}\right|_{\partial \Omega}=g, \\
\partial_{t} u & =-\Delta \tilde{z}, & & \left.u\right|_{\partial \Omega}=u_{D}, \\
\partial_{t} n_{p} & =\delta \nabla \tilde{z}+\Delta n_{p}-\mu n_{p}, & & \left.n_{p}\right|_{\partial \Omega}=n_{D, p}, \\
\partial_{t} n_{3} & =\Delta n_{3}-\mu n_{3}, & & \left.n_{3}\right|_{\partial \Omega}=n_{D, 3}, \\
|n|^{2}-1 & =0, & & \text { a.e. in } \Omega .
\end{aligned}
$$

The essential difference with respect to the model proposed by Uchida [32], and analyzed in [6] for a rigid constraint $|n|=1$, is the additional derivative of $\operatorname{div} n_{p}$ in the coupling term $\tilde{z}$. This leads to additional difficulties in the stability analysis of the numerical scheme as compared to [6]. We propose in Section 3.3 a semi-implicit algorithm for the computation of approximate solutions in finite element spaces and prove uniform bounds for a suitable energy of the system. We then present in Section 4 several numerical experiments displaying quite interesting dynamics of defects. Note, that the $L^{2}$-gradient flow for biomembranes is obtained by adding the term $\frac{1}{\varepsilon^{2}}\left(\left|n_{3}\right|^{2}-\xi_{0}^{2}\right) n_{3}$ to the equation (1.8).

\subsection{Qualitative analysis of defect-shape interaction}

In order to understand the interaction of defects and shape in the biomembrane case, i.e., when the angle between the director and surface normal is fixed, we consider in Sections 2 and 4 a decomposition of the director field $n$ into a tangential and normal part. The normal part is a fixed multiple of the surface normal and the tangential, planar part $n_{p}$ has a fixed length. This decomposition allows us to construct in Section 2 formal stationary solutions with $-\Delta u=\operatorname{div} n_{p}$. The proposed director fields are (infinite energy) limits of energy-minimizing configurations for a Ginzburg-Landau regularization of the Frank energy $\int_{\Omega}\left|\nabla n_{p}\right|^{2} \mathrm{~d} x$ subject to their own boundary data, cf. [8]. This approach allows a precise characterization of the shape corresponding to different defects and provides insight on the long time asymptotics of $(u, n)$. In the numerical experiments for the linear model on graphs reported in Section 4 we allow the tangential part of the director field to develop an out-of-plane component, so that the full director field violates the angle condition and finite energy minimizers are possible. We observe that for defects of degree \pm 1 the asymptotic behavior is dictated by the solutions found in Section 2. It is important to realize that, in contrast to [6], our new model with rigid constraint $|n|=1$ admits defects in the limit because $n_{p}$ is allowed to go out of plane near point singularities, a feature fully documented in Section 4 . The numerical results for the full model on closed surfaces reported in Section 6 show that the theoretical and practical predictions of the interaction of defects with the membrane shape in the simplified case explain the interesting dynamics occurring in the full biomembrane model for which the presence of defects is unavoidable if the angle between $v$ and $n$ is fixed. 


\subsection{Nonlinear model on closed surfaces}

For a smooth embedded surface $\Gamma \subset \mathbb{R}^{3}$, a director field $n: \Gamma \rightarrow \mathbb{S}^{2}$ and constants $\delta, \varepsilon$ and $\lambda$, we consider the energy (1.2) augmented as follows

$$
\begin{aligned}
E(\Gamma, n)=\frac{1}{2} \int_{\Gamma}\left|\operatorname{div}_{\Gamma} v-\delta \operatorname{div}_{\Gamma} n\right|^{2} \mathrm{~d} \sigma+\frac{\lambda}{2} & \int_{\Gamma}\left|\nabla_{\Gamma} n\right|^{2} \mathrm{~d} \sigma \\
& +\frac{1}{2} \int_{\Gamma} \mu\left(|n|^{2}-1\right) \mathrm{d} \sigma+\frac{1}{2 \varepsilon^{2}} \int_{\Gamma} f(n \cdot v) \mathrm{d} \sigma,
\end{aligned}
$$

where $\mu$ is the Lagrange multiplier for the rigid constraint $|n|=1$ and $f$ is given by $f(x)=$ $\left(x^{2}-\xi_{0}^{2}\right)^{2}$, for $\xi_{0} \in[0,1]$. The last term penalizes the deviation of the three-dimensional director field $n$ from the cone of all vectors that have a given angle with respect to the unit normal $v$ to the surface, as discussed already in Section 1.3. Thus, for $\varepsilon=\infty$, which corresponds to neglecting the last term, we obtain the surfactant case, while $\varepsilon \ll 1$ results in the modelling of biomembranes. In Section 5 , we derive a variation of the energy with respect to $\Gamma$ and $n$, which is the first step on the way to discover critical points of $E(\Gamma, n)$. We also introduce a semi-implicit algorithm based on parametric finite elements of Barrett, Garcke and Nünberg [3] to model the $L^{2}$-gradient flow of $E(\Gamma, n)$, see also [2]. As we are interested in the simulation of cells and biomembranes, side conditions like conservation of the enclosed volume and/or the surface area are important. For this purpose we use a Newton-iteration method, as proposed in [9]. In Section 6 we explore the behavior of the nonlinear model via simulations. We first show that for $\delta=1$ and without angle penalization, the vectors $v$ and $n$ tend to align since this minimizes $\left(\operatorname{div}_{\Gamma}(v-n)\right)^{2}$. We also display the dynamics of defects of degree \pm 1 and observe that locally the membrane shape is similar to that discovered earlier in the graph case. We conclude that defects of the director field $n$ have a dramatic effect on the shape of $\Gamma$, as observed in experiments, e.g., reported in [24].

\section{Qualitative Behavior of Graphs}

To build intuition about the mechanisms introduced by the coupling term in the model, we fix stationary tangential director fields $n_{p}$ of unit length and compute a function $u \in H_{0}^{1}(\Omega)$ for which the first term in (1.2) vanishes. For ease of readability we omit the subscript $p$ throughout this section. We thus impose that the auxiliary variable

$$
\tilde{z}=\Delta u+\operatorname{div} n
$$

vanishes, thereby giving the relation

$$
-\Delta u=\operatorname{div} n .
$$

Motivated by experimental observations we are particularly concerned with the surface structure when the director field represents a defect of positive or negative degree-one, i.e.,

$$
n=\exp ( \pm i \theta)=\cos \theta \pm i \sin \theta
$$

in polar coordinates $(r, \theta)$ and complex notation. Notice that for such a field $n$ we have $\int_{\Omega}|\nabla n|^{2} \mathrm{~d} x=\infty$, so $n$ cannot be a minimizer of the energy which involves the Dirichlet integral of $n$, but it arises as the limit of minimizers of a corresponding Ginzburg-Landau regularization that penalizes the unit-length constraint, cf. [8]. Therefore our calculations are only meant to explain the 
structures observed in our experiments of Section 4 which necessarily involve regularizations of the corresponding fields. We first compute the divergence

$$
\operatorname{div} n=\partial_{x} \cos \theta \pm \partial_{y} \sin \theta=-\sin \theta \partial_{x} \theta \pm \cos \theta \partial_{y} \theta
$$

and recall that $\theta=\arctan y / x$, whence

$$
\partial_{x} \theta=\frac{-y}{x^{2}+y^{2}}, \quad \partial_{y} \theta=\frac{x}{x^{2}+y^{2}} .
$$

We insert this result into the expression for $\operatorname{div} n$ and obtain

$$
\operatorname{div} n=\frac{y^{2} \pm x^{2}}{r^{3}}=\frac{\sin ^{2} \theta \pm \cos ^{2} \theta}{r} .
$$

\subsection{Positive degree-one defects}

We now take $n=\exp (i \theta)=\cos \theta+i \sin \theta$. We thus seek $u$ such that the inhomogeneous equation (in polar coordinates)

$$
\Delta u=\frac{1}{r} \partial_{r}\left(r \partial_{r} u\right)+\frac{1}{r^{2}} \partial_{\theta}^{2} u=-\frac{1}{r}
$$

holds. It is natural to look for a radial solution $u(r)=-r^{\alpha}$ and the expression for $\Delta u$ implies the necessary condition $\alpha=1$ and the cone-like surface (see Figure 1, top):

$$
u(r)=-r .
$$

Consider now the director field $n=e^{i(\theta+\pi / 2)}$ rotated by an angle $\pi / 2$. Such an $n$ satisfies $\operatorname{div} n=$ 0 , whence $u=0$; this is depicted in the lower plot of Figure 1. Any other rotation $n=e^{i\left(\theta+\theta_{0}\right)}$ by an angle $\theta_{0}$ can be expressed as $n=\cos \theta_{0} n_{1}+\sin \theta_{0} n_{2}$ with $n_{1}, n_{2}$ the director fields in Figure 1. The corresponding solution is thus

$$
u=-r \cos \theta_{0}
$$

\subsection{Negative degree-one defects}

We now take $n=\exp (-i \theta)=\cos \theta-i \sin \theta$. We thus seek $u$ as a solution of the inhomogeneous equation

$$
\Delta u=\frac{1}{r} \partial_{r}\left(r \partial_{r} u\right)+\frac{1}{r^{2}} \partial_{\theta}^{2} u=-\frac{\sin ^{2} \theta-\cos ^{2} \theta}{r}=-\frac{\cos (2 \theta)}{r} .
$$

We try a solution of the form $u(r, \theta)=C r^{\alpha} \cos (2 \theta)$ for suitable constants $C, \alpha$ and evaluate the partial differential equation to obtain the necessary condition

$$
\Delta u=C\left(\alpha^{2}-4\right) r^{\alpha-2} \cos (2 \theta)=-\frac{\cos (2 \theta)}{r},
$$

whence $\alpha=1, C=1 / 3$ and

$$
u(r, \theta)=\frac{1}{3} r \cos (2 \theta)
$$



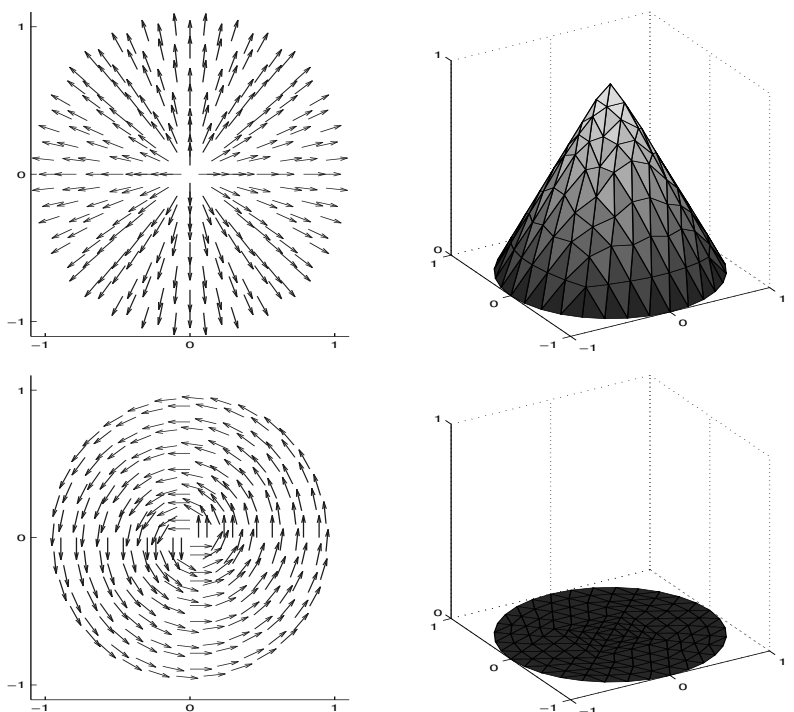

FIG. 1. Positive degree-one defect: director field $n=e^{i \theta}$ and cone-like surface $u=1-r$ (top), and rotated director field $n=e^{i(\theta+\pi / 2)}$ and function $u=0$ (bottom) related by (finite element discretizations of) $-\Delta u=\operatorname{div} n$ with $\left.u\right|_{\partial \Omega}=0$.

This solution is a saddle and is depicted in Figure 2 (top). Consider now the director field $n=$ $e^{-i\left(\theta-\theta_{0}\right)}$ which can be written as $n=e^{-i\left(\theta-\theta_{0} / 2\right)} e^{i \theta_{0} / 2}$. We thus realize that the value of $n$ at $\theta$ results from reading the value at $\theta-\theta_{0} / 2$ and rotating clockwise by $\theta_{0} / 2$, an effective rotation of $e^{-i \theta}$ by the angle $\theta_{0} / 2$. The corresponding solution thus reads

$$
u(r, \theta)=\frac{1}{3} r \cos \left(2 \theta-\theta_{0}\right) .
$$

Figure 2 (bottom) displays such a pair $(u, n)$ for $\theta_{0}=\pi / 2$.

\section{A semi-implicit scheme for graphs}

For simplicity we suppress in this section the index $h$ in connection with all finite element spaces and functions, that is, we write, e.g., $\mathcal{J}, \mathbb{V}$ and $(u, n)$ instead of $\mathcal{J}_{h}, \mathbb{V}_{h}$ and $\left(u_{h}, n_{h}\right)$, respectively. We use upper indices for the functions at discrete time steps. In particular $n^{0} \in \mathbb{V}$ is a suitable approximation of the initial data $n_{D}$. We point out that the definition and the analysis of the $L^{2}$ gradient flow presented here is for surfactants. The linearized model for biomembranes, which will be experimentally investigated in Section 4, is obtained by adding a lower order term that can be neglected in the analysis.

\subsection{Finite element spaces}

We let $\tau$ be a regular triangulation [11] of $\Omega$ into triangles of maximal diameter $h>0$. We denote by $\mathbb{V}=\mathbb{V}(\mathcal{J})$ the space of all continuous functions on $\Omega$ that are affine on the elements in the triangulation $\mathcal{J}$ and we set $\mathbb{V}_{0}=\mathbb{V} \cap H_{0}^{1}(\Omega)$. We say that $\mathcal{J}$ is weakly acute if the sum of every 

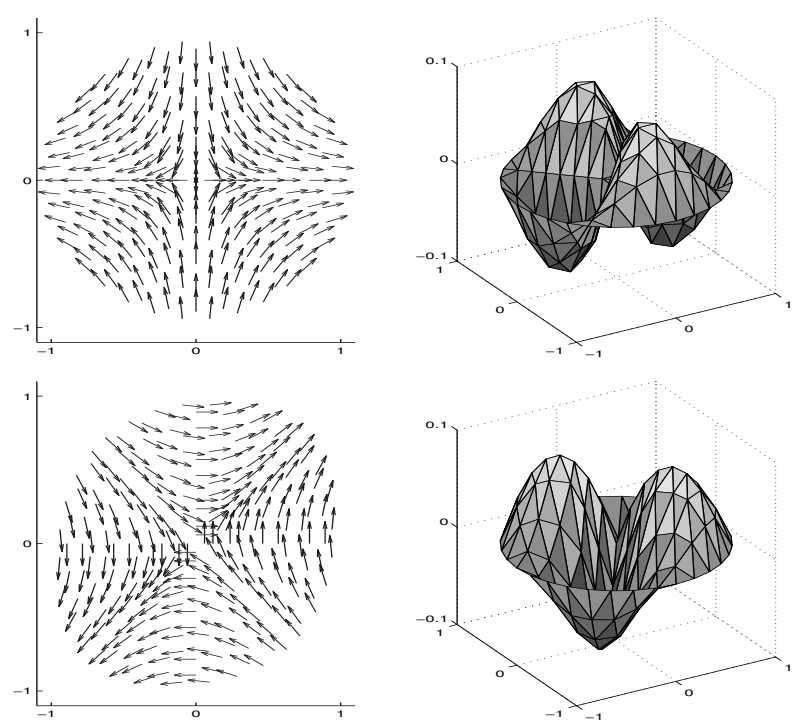

FIG. 2. Negative degree-one defect: Director field $n=e^{-i \theta}$ and saddle-like surface $u(r, \theta) \approx \frac{1}{3} r \cos (2 \theta)$ (top), and rotated director field $n=e^{-i(\theta-\pi / 2)}$ and corresponding rotated saddle-like surface $u(r, \theta) \approx \frac{1}{3} r \cos (2 \theta-\pi / 2)$ (bottom) related by (finite element discretizations of) $-\Delta u=\operatorname{div} n$ and $\left.u\right|_{\partial \Omega}=0$. Due to the boundary condition $u$ mimics the exact saddle structure only in a neighborhood of the origin.

pair of angles opposite to an interior edge is bounded by $\pi$ and if the angle opposite to every edge on the boundary is less than or equal to $\pi / 2$. Let $\left(\varphi_{a}\right)_{a \in n}$ denote the standard nodal basis of $\mathbb{V}$. For later purposes, we note that if $\mathcal{J}$ is weakly acute then [5]

$$
K_{i, j}:=\int_{\Omega} \nabla \varphi_{a_{i}} \cdot \nabla \varphi_{a_{j}} \mathrm{~d} x \leqslant 0 \quad \text { for all } a_{i} \neq a_{j} \in \mathfrak{n}
$$

where $\eta=\left\{a_{1}, \ldots, a_{N}\right\}$ denotes the set of nodes in $\mathcal{J}$. For completeness we include now a monotonicity estimate due to Bartels [5] for finite element methods, following the seminal work of Alouges [1].

LEMmA 3.1 (monotonicity) Let $\mathcal{J}$ be weakly acute, and let $\widetilde{n} \in \mathbb{V}^{3}$ be such that $|\widetilde{n}(a)| \geqslant 1$ for all $a \in \eta$, and define $n \in \mathbb{V}^{3}$ by setting $n(a)=\widetilde{n}(a) / \widetilde{n}(a) \mid$ for all $a \in \eta$. Then

$$
\|\nabla n\| \leqslant\|\nabla \widetilde{n}\| .
$$

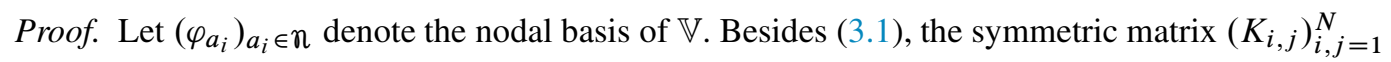


satisfies $\sum_{j=1}^{N} K_{i, j}=0$ owing to $\sum_{j=1}^{N} \varphi_{a_{j}}=1$. We observe the relations

$$
\begin{aligned}
\|\nabla n\|^{2} & =\sum_{i, j=1}^{N} K_{i, j} n\left(a_{i}\right) \cdot n\left(a_{j}\right) \\
& =\frac{1}{2} \sum_{i, j=1}^{N} K_{i, j} n\left(a_{i}\right) \cdot\left(n\left(a_{j}\right)-n\left(a_{i}\right)\right)+\frac{1}{2} \sum_{i, j=1}^{N} K_{i, j} n\left(a_{j}\right) \cdot\left(n\left(a_{i}\right)-n\left(a_{j}\right)\right) \\
& =-\frac{1}{2} \sum_{i, j=1}^{N} K_{i, j}\left|n\left(a_{i}\right)-n\left(a_{j}\right)\right|^{2} .
\end{aligned}
$$

The assertion is proved if $\left|n\left(a_{i}\right)-n\left(a_{j}\right)\right|^{2} \leqslant\left|\widetilde{n}\left(a_{i}\right)-\widetilde{n}\left(a_{j}\right)\right|^{2}$ for all $i, j=1, \cdots, N$. Hence, it suffices to show $\left|\frac{a}{|a|}-\frac{b}{|b|}\right| \leqslant|a-b|$, for $a, b \in \mathbb{R}^{3}$ with $|a|,|b| \geqslant 1$. This follows from the Lipschitz continuity of $\pi_{\mathbb{S}^{2}}:\left\{x \in \mathbb{R}^{3}:|x| \geqslant 1\right\} \rightarrow \mathbb{S}^{2}, x \mapsto x /|x|$.

For a fixed time-step size $\tau>0$ let $t_{j}=j \tau$ for all $j \geqslant 0$. Given $q \in[\mathbb{V}]^{3}$ we define the space of tangential updates with respect to the sphere for a given vector field $q$ with $|q(a)|=1$ for all $a \in \eta$ by

$$
\mathcal{F}[q]=\left\{r \in\left[\mathbb{V}_{0}\right]^{3}: r(a) \cdot q(a)=0 \quad \text { for all } a \in \mathfrak{n}\right\} .
$$

Since we use time-independent boundary conditions we may assume that we are given approximations $n^{0} \in[\mathbb{V}]^{3}$ and $u^{0} \in \mathbb{V}$ of $n_{D}$ and $u_{D}$ with $\left|n^{0}(a)\right|=1$ for all $a \in \eta$. Moreover we replace the additional variable $\tilde{z}=\Delta u+\delta \operatorname{div} n_{p}$, which has a Dirichlet boundary value $g$, by $z=\tilde{z}-g$, which has vanishing trace. Given $n^{0} \in[\mathbb{V}]^{3}$ and $u^{0} \in \mathbb{V}$, we let $z^{0} \in \mathbb{V}_{0}$ be an approximation to $z(0)$ defined as

$$
\left(z^{0}, y\right)=-(g, y)-\left(\nabla u^{0}, \nabla y\right)-\delta\left(n_{p}^{0}, \nabla y\right) \quad \text { for all } y \in \mathbb{V}_{0},
$$

and observe that the right-hand side in this equality defines a continuous linear form on $\mathbb{V}$. Since the $L^{2}$ inner product is a norm on $\mathbb{V}_{0}$ (with zero Dirichlet conditions), existence of a unique solution $z^{0}$ follows from the Lax-Milgram lemma.

In the numerical analysis of our proposed scheme we will need to control $\left\|\nabla z^{0}\right\|$. For this we assume for simplicity that $\left.\Delta u^{0}\right|_{\partial \Omega}=0$. Then, we define the discrete Laplacian $\Delta^{0}$ with zero boundary values for a finite element function $v$ to be the unique element $\Delta^{0} v \in \mathbb{V}_{0}$ that satisfies

$$
\left(\Delta^{0} v, w\right)=-(\nabla v, \nabla w) \text { for all } w \in \mathbb{V}_{0}
$$

and let $\Pi^{0}$ denote the $L^{2}$ projection onto $\mathbb{V}_{0}$. We then have that

$$
z^{0}=-\Pi^{0}\left(g-\delta \operatorname{div} n_{p}^{0}\right)+\Delta^{0} u^{0}
$$

and

$$
\left\|\nabla z^{0}\right\| \leqslant\left\|\nabla\left[-\Pi^{0}\left(g-\delta \operatorname{div} n_{p}^{0}\right)+\Delta^{0} u^{0}\right]\right\| .
$$

The assumption $\left.\Delta u^{0}\right|_{\partial \Omega}=0$ can be avoided by appropriately splitting $g=\left.\Delta u(0)\right|_{\partial \Omega}+\tilde{g}$ and replacing $g$ by $\tilde{g}$ in the foregoing discussion. 


\subsection{Difference quotients}

We use two definitions of difference quotients in time, i.e., for any sequence $\left(u^{j}\right)$ and for a sequence $\left(n^{j}\right)$ which is obtained by a post processing of a sequence $\left(\widetilde{n}^{j}\right)$ we write

$$
\mathrm{d}_{t} u^{j}=\frac{1}{\tau}\left(u^{j}-u^{j-1}\right), \quad \widetilde{\mathrm{d}}_{t} n^{j}=\frac{1}{\tau}\left(\widetilde{n}^{j}-n^{j-1}\right) .
$$

For all $j \in \mathbb{N}, j \geqslant 1$, we have

$$
\begin{aligned}
& \left(\mathrm{d}_{t} u^{j}, u^{j}\right)=\frac{1}{2 \tau}\left(\left\|u^{j}\right\|^{2}-\left\|u^{j-1}\right\|^{2}\right)+\frac{\tau}{2}\left\|\mathrm{~d}_{t} u^{j}\right\|^{2}=\frac{1}{2} \mathrm{~d}_{t}\left\|u^{j}\right\|^{2}+\frac{\tau}{2}\left\|\mathrm{~d}_{t} u^{j}\right\|^{2}, \\
& \left(\widetilde{\mathrm{d}}_{t} n^{j}, \widetilde{n}^{j}\right)=\frac{1}{2 \tau}\left(\left\|\widetilde{n}^{j}\right\|^{2}-\left\|n^{j-1}\right\|^{2}\right)+\frac{\tau}{2}\left\|\widetilde{\mathrm{d}}_{t} n^{j}\right\|^{2}=\frac{1}{2} \widetilde{\mathrm{d}}_{t}\left\|n^{j}\right\|^{2}+\frac{\tau}{2}\left\|\widetilde{\mathrm{d}}_{t} n^{j}\right\|^{2} .
\end{aligned}
$$

We recall a useful estimate for the discrepancy $\widetilde{n}^{j}-n^{j}$, and include a short proof [6, Proposition 4.2].

LEMMA 3.2 (Discrepancy $\left\|\widetilde{n}^{j}-n^{j}\right\|$ ) For the sequences $\left\{\widetilde{n}^{j}\right\}_{j \geqslant 0}$ and $\left\{n^{j}\right\}_{j \geqslant 0}$ constructed by the numerical scheme in Section 3.3 below, that is, satisfying in particular the orthogonality condition $\left(n^{j-1}, \tilde{n}^{j}-n^{j-1}\right)=0$ for all $j \geqslant 0$, where we set $n^{-1}=0$, the following estimate holds for all $j \geqslant 1$,

$$
\left\|\widetilde{n}^{j}-n^{j}\right\|^{2} \leqslant \gamma_{0} \tau^{4}\left\|\widetilde{\mathrm{d}}_{t} n^{j}\right\|^{2}\left\|\nabla \widetilde{\mathrm{d}}_{t} n^{j}\right\|^{2}
$$

A possible numerical value for $\gamma_{0}$ is $\gamma_{0}=25$.

Proof. For all nodes $a \in \eta$, we can write

$$
\left|\widetilde{n}^{j}(a)-n^{j}(a)\right|=\left|\widetilde{n}^{j}(a)-\frac{\widetilde{n}^{j}(a)}{\left|\widetilde{n}^{j}(a)\right|}\right|=\left|\widetilde{n}^{j}(a)\right|-1 .
$$

Since $\widetilde{n}^{j}(a)=n^{j-1}(a)+\tau \widetilde{\mathrm{d}}_{t} n^{j}(a)$ we obtain from orthogonality, the normalization of $n^{j-1}$, and the estimate $\left(1+x^{2}\right)^{1 / 2} \leqslant 1+\frac{1}{2} x^{2}$ that

$$
\left|\widetilde{n}^{j}(a)-n^{j}(a)\right| \leqslant\left(\left|n^{j-1}(a)\right|^{2}+\tau^{2}\left|\widetilde{\mathrm{d}}_{t} n^{j}(a)\right|^{2}\right)^{1 / 2}-1 \leqslant \frac{\tau^{2}}{2}\left|\widetilde{\mathrm{d}}_{t} n^{j}(a)\right|^{2} .
$$

In view of standard estimates for mass-lumping we get the inequality $\left\|\widetilde{n}^{j}-n^{j}\right\|_{L^{2}(T)}^{2} \leqslant$ $\tau^{4}\left\|\widetilde{\mathrm{d}}_{t} n^{j}\right\|_{L^{4}(T)}^{4}$. We take the sum over all triangles, recall the fixed boundary values for $n^{j}$ and use the multiplicative interpolation estimate [25] to obtain

$$
\left\|\widetilde{n}^{j}-n^{j}\right\|_{L^{2}(\Omega)}^{2} \leqslant \tau^{4}\left\|\widetilde{\mathrm{d}}_{t} n^{j}\right\|_{L^{4}(\Omega)}^{4} \leqslant 25 \tau^{4}\left\|\widetilde{\mathrm{d}}_{t} n^{j}\right\|_{L^{2}(\Omega)}^{2}\left\|\nabla \widetilde{\mathrm{d}}_{t} n^{j}\right\|_{L^{2}(\Omega)}^{2} .
$$

This estimate concludes the proof.

We finally recall a variant of discrete integration in time: for $1 \leqslant j \leqslant J$ we have $\mathrm{d}_{t}\left(u^{j} v^{j}\right)=$ $\mathrm{d}_{t} u^{j} v^{j}+u^{j-1} \mathrm{~d}_{t} v^{j}$, whence for $1 \leqslant \ell \leqslant J$

$$
u^{J} v^{J}-u^{\ell-1} v^{\ell-1}=\tau \sum_{j=\ell}^{J} \mathrm{~d}_{t}\left(u^{j} v^{j}\right)=\tau \sum_{j=\ell}^{J} \mathrm{~d}_{t} u^{j} v^{j}+\tau \sum_{j=\ell}^{J} u^{j-1} \mathrm{~d}_{t} v^{j}
$$




\section{$3.3 \quad$ Numerical scheme}

We propose a semi-implicit method for (1.4) in which the computation of the director field is naturally decoupled from the calculation of $u^{j}$ and $z^{j}$. We set $\widetilde{n}^{0}=n^{0}$ and seek for $j \geqslant 1$ and given $u^{j-1}, \widetilde{n}^{j-1}, n^{j-1}$ functions

$$
u^{j} \in \mathbb{V}_{0}, \quad z^{j} \in \mathbb{V}_{0}, \quad \widetilde{\mathrm{d}}_{t} n^{j} \in \mathcal{F}\left[n^{j-1}\right]
$$

such that

$$
\begin{aligned}
\left(z^{j}, y\right)+\left(\nabla u^{j}, \nabla y\right)+\delta\left(\widetilde{n}_{p}^{j-1}, \nabla y\right) & =-(g, y) & & \text { for all } y \in \mathbb{V}_{0}, \\
\left(\mathrm{~d}_{t} u^{j}, v\right)-\left(\nabla z^{j}, \nabla v\right) & =(\nabla g, \nabla v) & & \text { for all } v \in \mathbb{V}_{0}, \\
\left(\widetilde{\mathrm{d}}_{t} n^{j}, m\right)-\delta\left(\nabla z^{j}, m_{p}\right)+\left(\nabla \widetilde{n}^{j}, \nabla m\right) & =\delta\left(\nabla g, m_{p}\right) & & \text { for all } m \in \mathcal{F}\left[n^{j-1}\right],
\end{aligned}
$$

where $\widetilde{n}^{j}=n^{j-1}+\tau \widetilde{\mathrm{d}}_{t} n^{j}$. Now set

$$
n^{j}(a)=\frac{\widetilde{n}^{j}(a)}{\left|\widetilde{n}^{j}(a)\right|}=\frac{n^{j-1}(a)+\tau \widetilde{\mathrm{d}}_{t} n^{j}(a)}{\left|n^{j-1}(a)+\tau \widetilde{\mathrm{d}}_{t} n^{j}(a)\right|}, \quad \text { for all } a \in \eta .
$$

We remark that the system (3.10)-(3.11) has the structure of a saddle-point problem, that is similar to a hybrid formulation of the bilaplacian (with penalty term), i.e., (3.10)-(3.11) can be rewritten as

$$
\begin{array}{rlr}
\left(z^{j}, y\right)+\left(\nabla u^{j}, \nabla y\right) & \left.=-(g, y)-\delta \widetilde{n}_{p}^{j-1}, \nabla y\right) & \text { for all } y \in \mathbb{V}_{0}, \\
\left(\nabla z^{j}, \nabla v\right)-\tau^{-1}\left(u^{j}, v\right) & =-\tau^{-1}\left(u^{j-1}, v\right)-(\nabla g, \nabla v) & \text { for all } v \in \mathbb{V}_{0} .
\end{array}
$$

Owing to the essential boundary conditions imposed on $z^{j}$ and $u^{j}$ a $P 1-P 1$ discretization is stable. The Lax-Milgram lemma implies the unique solvability of (3.12) on the non-empty linear space $\mathcal{F}\left[n^{j-1}\right]$.

\subsection{Stability analysis}

In this section we derive energy estimates for the solutions of the finite element discretization. In particular, we verify bounds (uniform in $\tau$ ) for the following quantities:

$$
\begin{aligned}
& A(J)=\frac{1}{4}\left\|\nabla z^{J}\right\|^{2}+\frac{1}{2} \sum_{j=1}^{J} \tau\left\|\mathrm{d}_{t} \nabla u^{j}\right\|^{2}+\frac{\tau}{2} \sum_{j=1}^{J} \tau\left\|\mathrm{d}_{t} \nabla z^{j}\right\|^{2}, \\
& B(J)=\frac{1}{2}\left\|z^{J}\right\|^{2}+\frac{1}{2}\left\|\nabla n^{J}\right\|^{2}+\frac{1}{2} \sum_{j=1}^{J} \tau\left(\left\|\mathrm{d}_{t} u^{j}\right\|^{2}+\left\|\widetilde{\mathrm{d}}_{t} n^{j}\right\|^{2}\right) \\
&+\frac{\tau}{2} \sum_{j=1}^{J} \tau\left(\left\|\mathrm{d}_{t} z^{j}\right\|^{2}+\left\|\widetilde{\mathrm{d}}_{t} \nabla n^{j}\right\|^{2}\right),
\end{aligned}
$$

for all $J \geqslant 1$. The quantities which are quadratic in $\tau$ do not provide uniform estimates for the solutions. However, they are needed in order to control various terms that appear on the righthand sides of the following estimates. We state all bounds for $j \geqslant 1$ and use the conventions that 
$\sum_{j=\ell}^{k}(\ldots)=0$ and $\sup _{j=\ell, \ldots, k}(\ldots)=0$ whenever $k<\ell$. We set $\widetilde{n}^{-1}=\widetilde{n}^{0}=n^{0}$, whence $\mathrm{d}_{t} \widetilde{n}^{0}=0$, and recall the definition (3.4) of $z^{0} \in \mathbb{V}^{0}$. We assume

$$
u_{D} \in H^{3}(\Omega), \quad n_{D} \in H^{2}(\Omega), \quad g \in H^{2}(\Omega) .
$$

Combined with (3.4), this implies that $\left\|\nabla z^{0}\right\|$ is uniformly bounded with respect to $h$.

LEMMA 3.3 (first strong estimate) If (3.13) holds, then there exists a constant $\gamma_{0}$ such that for $J \geqslant 1$

$$
\begin{aligned}
A(J)=\frac{1}{4}\left\|\nabla z^{J}\right\|^{2}+\frac{1}{2} \sum_{j=1}^{J} \tau\left\|\mathrm{d}_{t} \nabla u^{j}\right\|^{2}+\frac{\tau}{2} \sum_{j=1}^{J} \tau\left\|\mathrm{d}_{t} \nabla z^{j}\right\|^{2} \\
\leqslant 2 \gamma_{0}^{2} \delta^{2} \sup _{j=3, \ldots, J}\left(\left\|\nabla \widetilde{n}^{j-2}\right\|^{2}+\left\|\nabla n^{j-3}\right\|^{2}\right) \sum_{j=3}^{J} \tau\left\|\widetilde{\mathrm{d}}_{t} n^{j-2}\right\|^{2} \\
+\delta^{2} \sum_{j=2}^{J} \tau\left\|\widetilde{\mathrm{d}}_{t} n_{p}^{j-1}\right\|^{2}+\left\|\nabla z^{0}\right\|^{2}+\frac{3}{2}\|\nabla g\|^{2} .
\end{aligned}
$$

Proof. Using (3.4) and the discrete time derivative of (3.10), we obtain that for $j \geqslant 1$

$$
\left(\mathrm{d}_{t} z^{j}, y\right)+\left(\mathrm{d}_{t} \nabla u^{j}, \nabla y\right)+\delta\left(\mathrm{d}_{t} \widetilde{n}_{p}^{j-1}, \nabla y\right)=0,
$$

because $g$ is time-independent. The choices $y=\mathrm{d}_{t} u^{j}$ in (3.14) and $v=\mathrm{d}_{t} z^{j}$ in (3.11) yield

$$
\frac{1}{2} \mathrm{~d}_{t}\left\|\nabla z^{j}\right\|^{2}+\frac{\tau}{2}\left\|\mathrm{~d}_{t} \nabla z^{j}\right\|^{2}+\left\|\mathrm{d}_{t} \nabla u^{j}\right\|^{2}=-\delta\left(\mathrm{d}_{t} \widetilde{n}_{p}^{j-1}, \mathrm{~d}_{t} \nabla u^{j}\right)-\left(\nabla g, \mathrm{~d}_{t} \nabla z^{j}\right),
$$

because of (3.6). Since (3.12) gives an estimate for $\widetilde{\mathrm{d}}_{t} n^{j}$, it is thus natural to rewrite the right-hand side of (3.15) as follows:

$$
\begin{aligned}
-\delta\left(\mathrm{d}_{t} \widetilde{n}_{p}^{j-1}, \mathrm{~d}_{t} \nabla u^{j}\right) & =-\delta\left(\widetilde{\mathrm{d}}_{t} n_{p}^{j-1}, \mathrm{~d}_{t} \nabla u^{j}\right)-\frac{\delta}{\tau}\left(n_{p}^{j-2}-\widetilde{n}_{p}^{j-2}, \mathrm{~d}_{t} \nabla u^{j}\right) \\
& \leqslant \frac{1}{2}\left\|\mathrm{~d}_{t} \nabla u^{j}\right\|^{2}+\delta^{2}\left\|\widetilde{\mathrm{d}}_{t} n_{p}^{j-1}\right\|^{2}+\frac{\delta^{2}}{\tau^{2}}\left\|\widetilde{n}_{p}^{j-2}-n_{p}^{j-2}\right\|^{2} .
\end{aligned}
$$

For $j=1,2$, the last term vanishes; for $j \geqslant 3$ we use the estimate (3.8) to infer that

$$
\left\|\widetilde{n}_{p}^{j-2}-n_{p}^{j-2}\right\|^{2} \leqslant 2 \gamma_{0}^{2} \tau^{2}\left(\left\|\nabla \widetilde{n}^{j-2}\right\|^{2}+\left\|\nabla n^{j-3}\right\|^{2}\right)\left\|\widetilde{\mathrm{d}}_{t} n^{j-2}\right\|^{2} .
$$

We take the sum in the foregoing estimates and multiply by $\tau$. Using (3.9) and the fact that $g$ is time-independent, the second term on the right-hand side of (3.15) reduces to

$$
-\sum_{j=1}^{J} \tau\left(\nabla g, \mathrm{~d}_{t} \nabla z^{j}\right)=-\left(\nabla z^{J}, \nabla g\right)+\left(\nabla z^{0}, \nabla g\right) \leqslant \frac{1}{4}\left\|\nabla z^{J}\right\|^{2}+\frac{1}{2}\left\|\nabla z^{0}\right\|^{2}+\frac{3}{2}\|\nabla g\|^{2} .
$$

This gives the asserted estimate. 
LEMMA 3.4 (second strong estimate) Suppose that (3.13) is valid and $\mathcal{J}$ is weakly acute, whence $\tau$ satisfies (3.1). Then the following bound holds for $J \geqslant 1$ :

$$
\begin{aligned}
B(J) \leqslant \frac{1}{2}\left\|z^{J}\right\|^{2} & +\frac{1}{2}\left\|\nabla \widetilde{n}^{J}\right\|^{2}+\frac{1}{2} \sum_{j=1}^{J} \tau\left(\left\|\mathrm{d}_{t} u^{j}\right\|^{2}+\left\|\widetilde{\mathrm{d}}_{t} n^{j}\right\|^{2}\right) \\
& +\frac{\tau}{2} \sum_{j=1}^{J} \tau\left(\left\|\mathrm{d}_{t} z^{j}\right\|^{2}+\left\|\widetilde{\mathrm{d}}_{t} \nabla n^{j}\right\|^{2}\right) \\
\leqslant & \frac{1}{2}\left\|z^{0}\right\|^{2}+\frac{1}{2}\left\|\nabla n^{0}\right\|^{2}+\delta^{2} T\|\nabla g\|^{2}+\frac{T}{2}\|\Delta g\|^{2} \\
+ & \delta^{2} \tau \sum_{j=2}^{J} \tau^{2}\left\|\mathrm{~d}_{t} \nabla z^{j}\right\|^{2}+\delta^{2} \tau\left\|\nabla z^{J}\right\|^{2} \\
& +2 \delta \gamma_{0} \tau^{1 / 2} \max _{j=1, \ldots, J}\left\|\nabla z^{j}\right\| \sum_{j=2}^{J}\left(\tau\left\|\widetilde{\mathrm{d}}_{t} n^{j-1}\right\|^{2}+\tau^{2}\left\|\widetilde{\mathrm{d}}_{t} \nabla n^{j-1}\right\|^{2}\right) .
\end{aligned}
$$

Proof. We use (3.14) with $y=z^{j}$, (3.11) with $v=\mathrm{d}_{t} u^{j}$, and (3.12) with $m=\widetilde{\mathrm{d}}_{t} n^{j}$ and employ

$$
\left(\nabla \widetilde{n}^{j}, \widetilde{\mathrm{d}}_{t} \nabla n^{j}\right)=\frac{\tau}{2}\left\|\nabla \widetilde{\mathrm{d}}_{t} n^{j}\right\|^{2}+\frac{1}{2 \tau}\left(\left\|\nabla \widetilde{n}^{j}\right\|^{2}-\left\|\nabla n^{j-1}\right\|^{2}\right)
$$

which is a variant of (3.7), to arrive at

$$
\begin{array}{r}
\frac{1}{2} \mathrm{~d}_{t}\left\|z^{j}\right\|^{2}+\frac{\tau}{2}\left\|\mathrm{~d}_{t} z^{j}\right\|^{2}+\frac{1}{2 \tau}\left(\left\|\nabla \widetilde{n}^{j}\right\|^{2}-\left\|\nabla n^{j-1}\right\|^{2}\right)+\frac{\tau}{2}\left\|\widetilde{\mathrm{d}}_{t} \nabla n^{j}\right\|^{2}+\left\|\widetilde{\mathrm{d}}_{t} n^{j}\right\|^{2}+\left\|\mathrm{d}_{t} u^{j}\right\|^{2} \\
=-\delta\left(\mathrm{d}_{t} \widetilde{n}_{p}^{j-1}, \nabla z^{j}\right)+\delta\left(\nabla z^{j}, \widetilde{\mathrm{d}}_{t} n_{p}^{j}\right)+\left(\mathrm{d}_{t} \nabla u^{j}, \nabla g\right)+\delta\left(\widetilde{\mathrm{d}}_{t} n_{p}^{j}, \nabla g\right) .
\end{array}
$$

For the first two terms on the right-hand side we have for $j \geqslant 1$

$$
\begin{aligned}
-\delta\left(\mathrm{d}_{t} \widetilde{n}_{p}^{j-1}, \nabla z^{j}\right)+\delta\left(\nabla z^{j}, \widetilde{\mathrm{d}}_{t} n_{p}^{j}\right) & =-\frac{\delta}{\tau}\left(\widetilde{n}_{p}^{j-1}-\widetilde{n}_{p}^{j-2}-\left(\widetilde{n}_{p}^{j}-n_{p}^{j-1}\right), \nabla z^{j}\right) \\
& =\delta \tau\left(\mathrm{d}_{t}^{2} \widetilde{n}_{p}^{j}, \nabla z^{j}\right)+\frac{\delta}{\tau}\left(\widetilde{n}_{p}^{j-1}-n_{p}^{j-1}, \nabla z^{j}\right) .
\end{aligned}
$$

We substitute (3.17) into (3.16), multiply the resulting expression by $\tau$, and sum it from $j=1$ to $J$. In view of (3.8) and the fact that $\widetilde{n}_{p}^{0}=n_{p}^{0}$, the second term on the right-hand side becomes

$$
\begin{aligned}
\delta \sum_{j=2}^{J}\left(\widetilde{n}_{p}^{j-1}-n_{p}^{j-1}, \nabla z^{j}\right) & \leqslant \delta \sum_{j=2}^{J}\left\|\widetilde{n}_{p}^{j-1}-n_{p}^{j-1}\right\|\left\|\nabla z^{j}\right\| \\
& \leqslant \delta \max _{j=2, \ldots, J}\left\|\nabla z^{j}\right\| \sum_{j=2}^{J} \gamma_{0} \tau^{2}\left\|\widetilde{\mathrm{d}}_{t} n^{j-1}\right\|\left\|\widetilde{\mathrm{d}}_{t} \nabla n^{j-1}\right\| \\
& \leqslant \frac{\delta}{2} \gamma_{0} \tau^{1 / 2} \max _{j=2, \ldots, J}\left\|\nabla z^{j}\right\| \sum_{j=2}^{J}\left(\tau\left\|\widetilde{\mathrm{d}}_{t} n^{j-1}\right\|^{2}+\tau^{2}\left\|\widetilde{\mathrm{d}}_{t} \nabla n^{j-1}\right\|^{2}\right) .
\end{aligned}
$$


Instead, for the first term on the right-hand side we use the partial summation formula (3.9), to deduce

$$
\begin{aligned}
\delta \tau \sum_{j=1}^{J} \tau\left(\mathrm{d}_{t}^{2} \widetilde{n}_{p}^{j}, \nabla z^{j}\right)= & \delta \tau \sum_{j=1}^{J} \tau\left(\mathrm{d}_{t}\left(\mathrm{~d}_{t} \widetilde{n}_{p}^{j}\right), \nabla z^{j}\right) \\
= & -\delta \tau \sum_{j=1}^{J} \tau\left(\mathrm{d}_{t} \widetilde{n}_{p}^{j-1}, \mathrm{~d}_{t} \nabla z^{j}\right)-\delta \tau\left(\mathrm{d}_{t} \widetilde{n}_{p}^{0}, \nabla z^{0}\right)+\delta \tau\left(\mathrm{d}_{t} \widetilde{n}_{p}^{J}, \nabla z^{J}\right) \\
= & -\delta \tau \sum_{j=2}^{J} \tau\left(\widetilde{\mathrm{d}}_{t} n_{p}^{j-1}, \mathrm{~d}_{t} \nabla z^{j}\right)-\delta \tau \sum_{j=2}^{J}\left(n_{p}^{j-2}-\widetilde{n}_{p}^{j-2}, \mathrm{~d}_{t} \nabla z^{j}\right) \\
& \quad+\delta \tau\left(\widetilde{\mathrm{d}}_{t} n_{p}^{J}, \nabla z^{J}\right)+\delta\left(n_{p}^{J-1}-\widetilde{n}_{p}^{J-1}, \nabla z^{J}\right)=I+I I+I I I+I V .
\end{aligned}
$$

We now examine terms $I-I V$ separately. For $I$ and $I I I$ we simply use Cauchy-Schwarz to write

$$
I \leqslant \frac{1}{4} \sum_{j=2}^{J} \tau\left\|\widetilde{\mathrm{d}}_{t} n_{p}^{j-1}\right\|^{2}+\delta^{2} \tau^{2} \sum_{j=2}^{J} \tau\left\|\mathrm{d}_{t} \nabla z^{j}\right\|^{2}, \quad I I \leqslant \frac{\tau}{4}\left\|\widetilde{\mathrm{d}}_{t} n_{p}^{J}\right\|^{2}+\delta^{2} \tau\left\|\nabla z^{J}\right\|^{2} .
$$

Since $\tau\left\|\mathrm{d}_{t} \nabla z^{j}\right\| \leqslant 2 \max _{1 \leqslant j \leqslant J}\left\|\nabla z^{j}\right\|$, we proceed as in (3.19) to find

$$
\begin{aligned}
I I & \leqslant \delta \tau \sum_{j=2}^{J}\left\|n_{p}^{j-2}-\widetilde{n}_{p}^{j-2}\right\|\left\|\mathrm{d}_{t} \nabla z^{j}\right\| \\
& \leqslant \delta \gamma_{0} \tau^{1 / 2} \max _{j=1, \ldots, J}\left\|\nabla z^{j}\right\| \sum_{j=1}^{J}\left(\tau\left\|\widetilde{\mathrm{d}}_{t} n^{j-2}\right\|^{2}+\tau^{2}\left\|\widetilde{\mathrm{d}}_{t} \nabla n^{j-2}\right\|\right)
\end{aligned}
$$

and

$$
I V \leqslant \delta\left\|n_{p}^{J-1}-\widetilde{n}_{p}^{J-1}\right\|\left\|\nabla z^{J}\right\| \leqslant \frac{\delta}{2} \gamma_{0} \tau^{1 / 2}\left\|\nabla z^{J}\right\|\left(\tau\left\|\widetilde{\mathrm{d}}_{t} n^{J-1}\right\|^{2}+\tau^{2}\left\|\widetilde{\mathrm{d}}_{t} \nabla n^{J-1}\right\|\right) .
$$

It remains to estimate the terms involving $g$ in the summation of (3.16). For the first term we use (3.13) and integrate by parts to get

$$
\sum_{j=1}^{J} \tau\left(\nabla g, \nabla \mathrm{d}_{t} u^{j}\right)=-\sum_{j=1}^{J} \tau\left(\Delta g, \mathrm{~d}_{t} u^{j}\right) \leqslant \frac{1}{2} \sum_{j=1}^{J} \tau\left\|\mathrm{d}_{t} u^{j}\right\|^{2}+\frac{T}{2}\|\Delta g\|^{2},
$$

where $T \geqslant \tau J$ is the final time. For the second term we simply use Cauchy-Schwarz

$$
\delta \sum_{j=1}^{J} \tau\left(\nabla g, \widetilde{\mathrm{d}}_{t} n_{p}^{j}\right) \leqslant \frac{1}{4} \sum_{j=1}^{J} \tau\left\|\widetilde{\mathrm{d}}_{t} n^{j}\right\|^{2}+\delta^{2} T\|\nabla g\|^{2} .
$$

We finally combine the foregoing estimates and use the monotonicity $\left\|\nabla n^{j}\right\| \leqslant\left\|\nabla \widetilde{n}^{j}\right\|$ for $j=$ $1,2, \ldots, J-1$, established in (3.2), as well as $\widetilde{n}^{0}=n^{0}$, to obtain the asserted estimate. 
THEOREM 3.5 (a priori estimates) Let (3.13) hold and $\mathcal{J}$ be weakly acute. We define

$$
\begin{gathered}
E(J)=\frac{1}{2}\left\|z^{J}\right\|^{2}+\frac{1}{2}\left\|\nabla n^{J}\right\|^{2}, \quad Z_{0}=\left\|\nabla z^{0}\right\|^{2}+\frac{3}{2}\|\nabla g\|^{2}, \\
G=\delta^{2} T\|\nabla g\|^{2}+\frac{T}{2}\|\Delta g\|^{2},
\end{gathered}
$$

and set for arbitrary $0<\varepsilon<1$

$$
\bar{B}=E(0)+G+2 \tau^{\varepsilon}, \quad \bar{A}=16 \gamma_{0}^{2} \delta^{2} \bar{B}^{2}+2 \delta^{2} \bar{B}+Z_{0} .
$$

Suppose finally that

$$
\tau^{1-\varepsilon} \leqslant \frac{1}{4 \delta^{2} \bar{A}}, \quad \tau^{1 / 2-\varepsilon} \leqslant \frac{1}{8 \delta \gamma_{0} \bar{A}^{1 / 2} \bar{B}} .
$$

Then for all $J \geqslant 1$ we have

$$
\begin{aligned}
& A(J)=\frac{1}{2}\left\|\nabla z^{J}\right\|^{2}+\frac{\tau}{2} \sum_{j=1}^{J} \tau\left\|\mathrm{d}_{t} \nabla z^{j}\right\|^{2}+\frac{1}{2} \sum_{j=1}^{J} \tau\left\|\mathrm{d}_{t} \nabla u^{j}\right\|^{2} \leqslant \bar{A} \\
& B(J)=E(J)+\sum_{j=1}^{J} \tau\left(\left\|\mathrm{d}_{t} u^{j}\right\|^{2}+\frac{1}{2}\left\|\widetilde{\mathrm{d}}_{t} n^{j}\right\|^{2}\right)+\frac{\tau}{2} \sum_{j=1}^{J} \tau\left(\left\|\mathrm{d}_{t} z^{j}\right\|^{2}+\left\|\widetilde{\mathrm{d}}_{t} \nabla n^{j}\right\|^{2}\right) \leqslant \bar{B} .
\end{aligned}
$$

Proof. We proceed by induction. For $J=1$ the estimates of Lemmas 3.3 and 3.4 imply

$$
A(1) \leqslant Z_{0} \leqslant \bar{A}, \quad B(1) \leqslant E(0) \leqslant \bar{B} .
$$

Suppose now that the assertion has been verified for $J-1 \geqslant 1$, i.e., for $j=1, \ldots, J-1$ we have $A(j) \leqslant \bar{A}$ and $B(j) \leqslant \bar{B}$. Lemma 3.3 and the definition of $\bar{A}$ imply

$$
A(J) \leqslant 16 \gamma_{0}^{2} \delta^{2} \sup _{j=0, \ldots, J-1} B(j) B(J-2)+2 \delta^{2} B(J-1)+Z_{0} \leqslant \bar{A} .
$$

Similarly, Lemma 3.4 gives the upper bound

$$
B(J) \leqslant E(0)+G+4 \delta^{2} \tau A(J)+8 \delta \gamma_{0} \tau^{1 / 2} \max _{j=1, \ldots, J} A(j)^{1 / 2} B(J-1) .
$$

The induction hypothesis $B(J-1) \leqslant \bar{B}$ and the conditions on $\tau$ lead to

$$
B(J) \leqslant E(0)+G+2 \tau^{\varepsilon}=\bar{B}
$$

which proves the assertion of the theorem.

Remark 3.1 (regularity of $g$ ). The $H^{2}$-regularity of $g$, assumed in (3.13), can be weakened to $g \in H^{1}(\Omega)$ at the expense of a more technical proof of Theorem 3.5. In fact, we avoid integration by parts in the first term involving $g$ in Lemma 3.4 and instead write

$$
\sum_{j=1}^{J} \tau\left(\nabla g, \mathrm{~d}_{t} \nabla u^{j}\right) \leqslant \frac{\xi}{2} \sum_{j=1}^{J} \tau\left\|\nabla \mathrm{d}_{t} u^{j}\right\|^{2}+\frac{1}{2 \xi} \sum_{j=1}^{J} \tau\|\nabla g\|^{2}
$$


for $\xi>0$ arbitrarily small. The recursion for $B(J)$ in the proof of Theorem 3.5 now becomes

$$
B(J) \leqslant E(0)+G+\xi A(J)+4 \delta^{2} \tau A(J)+8 \delta \gamma_{0} \tau^{1 / 2} A(J)^{1 / 2} B(J-1) .
$$

Replacing this bound into the recursion of $A(J)$ allows us to absorb the term $\xi A(J)$ for $\xi$ sufficiently small.

\subsection{Weak solution of (1.4)}

The a priori estimates of Theorem 3.5 allow us to establish the existence of a weak solution of the continuous $L^{2}$ flow that satisfies an energy inequality. We now state precisely the notion of solution already introduced in (1.4) and refer the reader to [6] for details about passing to the limit.

DEFINITION 3.6 (weak solution) Let $\Omega \subset \mathbb{R}^{2}$ be a bounded and convex Lipschitz domain and fix $T>0$. We call a pair $(u, n)$ a weak solution of (1.4) in the time interval $I=(0, T)$ if the following assertions are true:

(i) $\quad n \in H^{1}\left(I ; L^{2}\left(\Omega ; \mathbb{R}^{2}\right)\right) \cap L^{\infty}\left(I ; H^{1}\left(\Omega ; \mathbb{R}^{2}\right)\right), u \in H^{1}\left(I ; L^{2}(\Omega)\right) \cap L^{\infty}\left(I ; H^{2}(\Omega)\right)$;

(ii) $|n(t, x)|=1$ for almost every $(t, x) \in I \times \Omega$;

(iii) $n(0, \cdot)=n_{D}, u(0, \cdot)=u_{D}$ with $u_{D} \in H^{3}(\Omega)$ and $n_{D} \in H^{2}(\Omega)$;

(iv) $\left.n(t, \cdot)\right|_{\partial \Omega}=n_{D}$ and $\left.u(t, \cdot)\right|_{\partial \Omega}=u_{D}$ in the sense of traces for almost every $t \in I$;

(v) $\Delta u+\delta \operatorname{div} n_{p} \in L^{2}\left(I ; H^{1}(\Omega)\right)$ and satisfies for a.e. $t \in I$ that $\left.\left(\Delta u+\delta \operatorname{div} n_{p}\right)\right|_{\Gamma}=g$ with $g \in H^{2}(\Omega)$ given;

(vi) for all $(m, v) \in L^{2}\left(I ; H_{0}^{1}\left(\Omega ; \mathbb{R}^{2}\right)\right) \times L^{2}\left(I ; H^{2}(\Omega) \cap H_{0}^{1}(\Omega)\right)$ satisfying $m \cdot n=0$ almost everywhere in $I \times \Omega$ we have

$$
\begin{array}{r}
\int_{I}\left\{\left(\partial_{t} u, v\right)+\left(\Delta u+\delta \operatorname{div} n_{p}, \Delta v\right)\right\} \mathrm{d} t-\int_{\partial \Omega} g \partial_{\nu} v \mathrm{~d} S=0, \\
\int_{I}\left\{\left(\partial_{t} n, m\right)+\left(\Delta u+\delta \operatorname{div} n_{p}, \delta \operatorname{div} m_{p}\right)+(\nabla n, \nabla m)\right\} \mathrm{d} t=0 .
\end{array}
$$

Remark 3.2. We note that the formulation of Theorem 3.5 allows one to deduce the existence of a solution $(u, n)$ that satisfies the energy inequality

$$
\begin{aligned}
\frac{1}{2}\left\|\Delta u+\delta \operatorname{div} n_{p}\right\|^{2}+ & \frac{1}{2}\|\nabla n\|^{2}+\int_{0}^{T}\left(\left\|\partial_{t} u\right\|^{2}+\frac{1}{2}\left\|\partial_{t} n\right\|^{2}\right) \mathrm{d} t \\
& \leqslant \frac{1}{2}\left\|\Delta u_{D}+\delta \operatorname{div}\left(n_{D}\right)_{p}\right\|^{2}+\frac{1}{2}\left\|\nabla n_{D}\right\|^{2}+\delta^{2} T\|\nabla g\|^{2}+\frac{T}{2}\|\Delta g\|^{2} .
\end{aligned}
$$

We refer the reader to [31] for related existence theories in the context of the harmonic map heat flow.

\section{Numerical experiments for graphs}

In this section we report on various numerical experiments for biomembranes carried out with the scheme devised and analyzed in the previous sections. Since we want to illustrate the interaction of defects and shape we consider the case of a membrane in the gel phase where the director field prefers to have a fixed angle with respect to the normal to the surface, say $\pi / 2$ for convenience. 
As in Section 2 the director field $n$ has unit length but is allowed to develop an out-of-plane component to accommodate for topological defects; we omit the index $p$ throughout this section for the tangential part $n_{p}$ of $n$. We thus augment the system of equations discussed in Section 3.4 by the term $\varepsilon^{-2}\left(\widetilde{n}_{3}^{j}, m_{3}\right)$, where the subscript 3 refers to the third, or out-of-plane, component of a vectorfield, in (3.12), i.e., for the evolution of the director field we employ the equation

$$
\left.\left(\widetilde{\mathrm{d}}_{t} n^{j}, m\right)-\delta\left(\nabla z^{j}, m_{p}\right)+\left(\nabla \widetilde{n}^{j}, \nabla m\right)+\varepsilon^{-2} \widetilde{n}_{3}^{j}, m_{3}\right)=0 .
$$

This modification corresponds to the additional penalty term

$$
\frac{1}{2 \varepsilon^{2}} \int_{\Omega}\left|n_{3}\right|^{2} \mathrm{~d} x
$$

in the energy, i.e., our energy functional is

$$
E(u, z, n)=\frac{1}{2} \int_{\Omega}|z|^{2} \mathrm{~d} x+\frac{1}{2} \int_{\Omega}|\nabla n|^{2} \mathrm{~d} x+\frac{1}{2 \varepsilon^{2}} \int_{\Omega}\left|n_{3}\right|^{2} \mathrm{~d} x
$$

subject to the relation $z=\Delta u+\delta \operatorname{div}\left(n_{1}, n_{2}\right)$, the pointwise constraint $|n|=1$, and Dirichlet boundary conditions for $u, z$, and $n$. We remark that the inclusion of an implicit treatment of the convex penalty term in the stability analysis for the numerical scheme in Section 3 poses no difficulties.

The goal of this section is to explore the qualitative behavior of the evolution for specific initial conditions with defects. Here, the terminology of a defect refers to a singularity in the renormalized planar part of the director field which is also called vortex. This evolution typically shows an initial phase with a significant change of the shape in order for the system to adjust to the given initial and boundary values which is followed by a slower evolution towards an equilibrium shape. In the figures we display typical intermediate shapes and states which are close to an equilibrium. In our simulations the domain $\Omega$ and the parameters $\delta, T$, and $\varepsilon$ are given by

$$
\Omega=(-1 / 2,1 / 2)^{2}, \quad \delta=1, \quad T=1, \quad \varepsilon=10^{-1} .
$$

We denote by $(r, \phi)$ the usual polar coordinates in $\mathbb{R}^{2}$ (with respect to the origin). The function $\varphi_{\varepsilon}$ which is used in the extension of a function given on $\partial \Omega$ to $\Omega$ is equal to $\varphi_{\varepsilon}(r)=\tanh (r / \varepsilon)$. The initial values are always chosen to be

$$
u_{0}=0, \quad g=\ell_{h}\left[\operatorname{div} n_{0}\right]
$$

for different choices of $n_{0}$ and where $\ell_{h}$ is the nodal interpolation operator. The sequence of triangulations $\tau_{\ell}$ is generated by $\ell$ uniform refinements (division of each triangle into four congruent ones) of the initial triangulation $\mathcal{J}_{0}$ of $\Omega$ which consists of two triangles obtained by dividing $\Omega$ along the diagonal $x_{1}=x_{2}$. Hence the mesh-size $h_{\ell}$ is given by $h_{\ell}=\sqrt{2} 2^{-\ell}$. Moreover we used $\tau_{\ell}=h_{\ell} /(8 \sqrt{2})$ as time-step size.

\subsection{Positive degree-one defect}

We choose boundary conditions which correspond to a defect of degree one, i.e.,

$$
\left.n_{0}\right|_{\partial \Omega}\left(x_{1}, x_{2}\right)=\left.n_{0}\right|_{\partial \Omega}(r \cos \phi, r \sin \phi)=(\cos (\phi), \sin (\phi), 0)=\left(e^{i \phi}, 0\right), \quad\left(x_{1}, x_{2}\right) \in \partial \Omega
$$


(in complex notation). Note that these boundary data do not allow for a continuous, purely planar extension, since any such extension would necessarily contain topological defects of infinite energy. Thus it is expected that the numerical solution will develop an out-of-plane component of the vectorfield despite the penalization of this component in the energy in order to accommodate the length constraint. For energetic reasons, there should be only one point in the domain where such a defect-like structure is observed. Therefore we define the extension of $n_{0}$ which is needed for the numerical scheme at all interior nodes $a \in \eta$ with the help of polar coordinates $\left(r_{2}, \phi_{2}\right)$ about $(-1 / 4,-1 / 4)$ by

$$
n_{0}(a)=n_{0}\left(r_{2} \cos \phi_{2}, r_{2} \sin \phi_{2}\right)=\left(\varphi_{\varepsilon}\left(r_{2}\right) \cos \left(\phi_{2}\right), \varphi_{\varepsilon}\left(r_{2}\right) \sin \left(\phi_{2}\right),\left(1-\varphi_{\varepsilon}\left(r_{2}\right)^{2}\right)^{1 / 2}\right) .
$$

Thus there is such a defect-like structure already present in the initial data but it is not located at the origin where it is expected to move during the evolution of the system.

The snapshots of the evolution in Figure 3 show indeed that this initial vortex moves slowly towards the origin, which is an energetically favorable configuration. At the same time, the surface develops a profile which is a smoothed version of the cone described in Section 2. The location of the maximal height moves together with the vortex towards the origin. The initially strong energy decay shown in the bottom plot of Figure 3 is related to the incompatibility of the initial data in the sense that $\Delta u_{0}+\delta \operatorname{div} n_{0}$ is large.

\subsection{Negative degree-one defect}

We employ

$$
\left.n_{0}\right|_{\partial \Omega}(r \cos \phi, r \sin \phi)=(\cos (-\phi), \sin (-\phi), 0)=\left(e^{-i \phi}, 0\right)
$$

with an extension to $\Omega$ so that the defect is located at $x=(-1 / 4,-1 / 4)$ as above. A defect of negative degree-one in the planar part of the director field is favored by the boundary conditions and already present in the extension of $\left.n_{0}\right|_{\partial \Omega}$ to $\Omega$. The corresponding evolution is shown in Figure 4 . As in the case of a positive degree-one defect we observe that the defect of negative degree-one moves towards the center of the domain. The surface adjusts to the defect by developing a saddleshape and follows its motion. Eventually we observe a stationary configuration with a saddle as predicted in Section 2. In accordance, the energy shows a rapid decay in the beginning and then only decreases moderately.

\subsection{Positive degree-two defect}

We set

$$
\left.n_{0}\right|_{\partial \Omega}(r \cos \phi, r \sin \phi)=(\cos (2 \phi), \sin (2 \phi), 0)=\left(e^{2 i \phi}, 0\right),
$$

with an extension to $\Omega$ as above so that the defect is located at the origin. This initial configuration of the director field is unstable as can be seen in the snapshots displayed in Figure 5. The vortex of degree two immediately splits into two defects of positive degree one and rotated by $\pm \pi / 2$ which subsequently repel each other and tend to maximize their distance. As is expected from the analysis in Section 2, the rotated defects do not induce local curvature and the observed shape corresponds to the nearly constant director field between the defects pointing in the negative $x_{1}$-direction. In this example we observe a rapid initial energy decay and another significant decay when the defects have reached a proper separation distance. 


\subsection{Two opposite degree-one defects}

With polar coordinates $\left(r_{ \pm}, \phi_{ \pm}\right)$about $( \pm 1 / 4,0)$ we set

$$
n_{0}(x)= \begin{cases}-\left(\cos \left(-\phi_{+}\right), \sin \left(-\phi_{+}\right), 0\right) & \text { for } x_{1}>0 \\ \left(\cos \left(\phi_{-}\right), \sin \left(\phi_{-}\right), 0\right) & \text { for } x_{1} \leqslant 0\end{cases}
$$

for $x=\left(x_{1}, x_{2}\right) \in \partial \Omega$, and extend to $\Omega$ as above so that the defects are located at $( \pm 1 / 4,0)$. We point out that in order to match smoothly the director field at $x_{1}=0$ the negative degree-one defect is that of Section 4.2 rotated by $\pi$. When two defects of opposite degree are present in the initial data, the surface adjusts with a shape as predicted in Section 2 by forming a cone and a saddle-like structure, cf. Figure 6 (middle). During the evolution, the vortices attract each other and eventually annihilate. The shape of the surface follows the location of the vortices. A smooth profile is still observable after the event of annihilation which is related to the fact that the boundary data for $n, z$ used in this example are non-constant. The energy curve shown in the bottom plot of Figure 6 reveals a rapid initial decay related to incompatible initial data, followed by a plateau corresponding to the motion of the vortices towards each other, and a strong decay when the annihilation of the vortices takes place.

\section{The nonlinear model on closed surfaces}

In this section we return to the nonlinear model of Section 1.6, which corresponds to the energy

$E(\Gamma, n):=\frac{1}{2} \int_{\Gamma}\left(\operatorname{div}_{\Gamma} v-\delta \operatorname{div}_{\Gamma} n\right)^{2} \mathrm{~d} \sigma+\frac{\lambda}{2} \int_{\Gamma}\left|\nabla_{\Gamma} n\right|^{2} \mathrm{~d} \sigma+\int_{\Gamma} \mu\left(|n|^{2}-1\right) \mathrm{d} \sigma+\frac{1}{2 \varepsilon^{2}} \int_{\Gamma} f(n \cdot v) \mathrm{d} \sigma$.

To formulate the gradient flow of $E(\Gamma, n)$ we need the first variation of $E(\Gamma, n)$. We thus start with a brief review of differential geometry which we next apply to derive the first variation in Section 5.2. We follow with a discretization of the gradient flow, using parametric finite elements and including constraints.

\subsection{Elementary differential geometry}

Let $U \subset \mathbb{R}^{2}$ be open and $X: U \rightarrow \mathbb{R}^{3},\left(u_{1}, u_{2}\right) \mapsto X\left(u_{1}, u_{2}\right)$ be a local parametrization of $\Gamma$. If $X_{i}=\partial_{u_{i}} X$, then the induced metric on $\Gamma$ is given by $g_{i j}=X_{i} \cdot X_{j}$. The inverse of $g_{i j}$ is $g^{i j}$ and the square root of the matrix $g^{i j}$ is $g_{i j}^{(-1 / 2)}$, i.e., $\sum_{k} g_{i k}^{(-1 / 2)} g_{j k}^{(-1 / 2)}=g^{i j}$. If $g=\operatorname{det}\left(g_{i j}\right)$, then the volume element on $\Gamma$ is given by $\mathrm{d} \sigma=\sqrt{g} \mathrm{~d} u_{1} \mathrm{~d} u_{2}$. The unit normal is $v=\frac{X_{1} \times X_{2}}{\left|X_{1} \times X_{2}\right|}$, and the second fundamental form is $h_{i j}=-\partial_{i} v \cdot X_{j}$. If $f$ and $F$ are scalar- and vector-valued functions on $\Gamma$ and $\tilde{f}, \tilde{F}$ are arbitrary extensions then the tangential gradient and divergence on $\Gamma$ are given by

$$
\nabla_{\Gamma} f=\nabla \tilde{f}-(v \cdot \nabla \tilde{f}) v, \quad \operatorname{div}_{\Gamma} F=\operatorname{div} \tilde{F}-v^{T} D \tilde{F} v .
$$

In the local coordinates defined above these operators are

$$
\left(\nabla_{\Gamma} f\right) \circ X=\sum_{i, j} g^{i j} \partial_{j}(f \circ X) X_{i}, \quad\left(\operatorname{div}_{\Gamma} F\right) \circ X=\sum_{i, j} g^{i j} \partial_{i}(F \circ X) \cdot X_{j}
$$



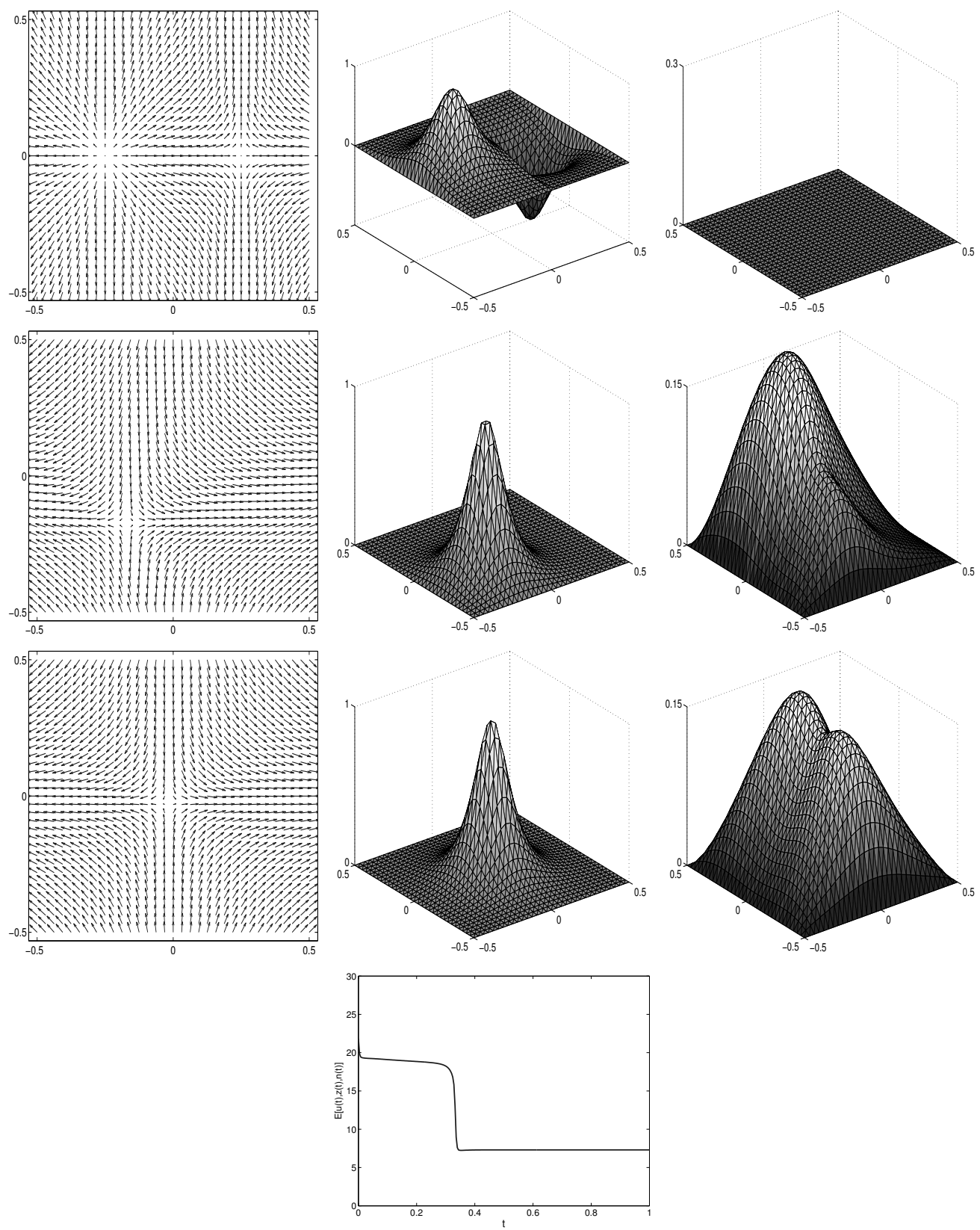

FIG. 3. Positive degree-one defect: in-plane component of the director field (left), out-of-plane component of the director field (middle), and height function (right) after $j=0,32,256$ time steps. The surface develops a smoothed out cone and follows the motion of the defect. The energy shows a rapid initial decay when the surface adjusts to the defect. 

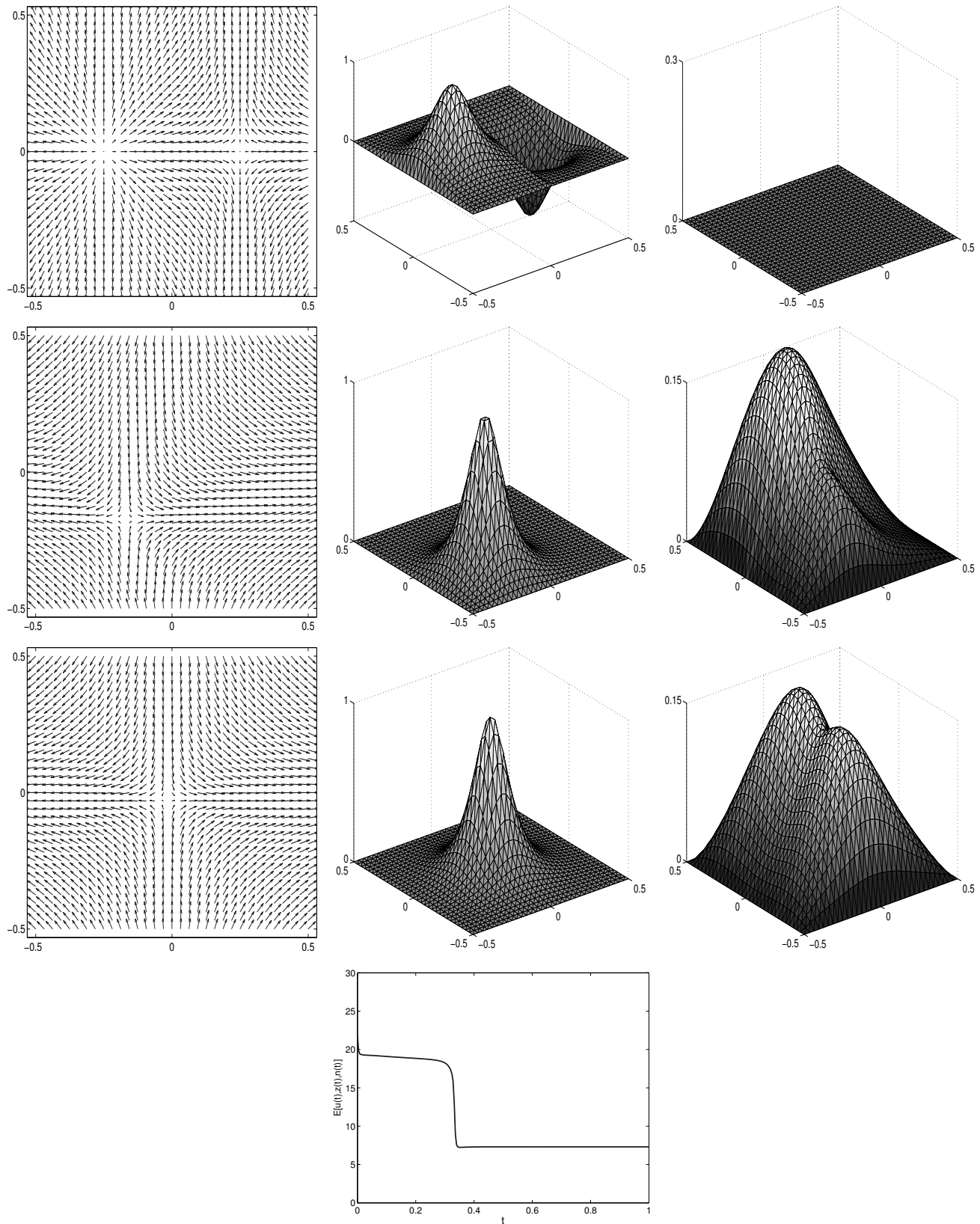

FIG. 4. Negative degree-one defect: in-plane component of the director field (left), out-of-plane component of the director field (middle), height function (right) after $j=0,8,16$ time steps. The height function shows a saddle shape in a neighborhood of the defect and this configuration is stable. The energy decays rapidly during the first time steps and remains almost constant subsequently. 

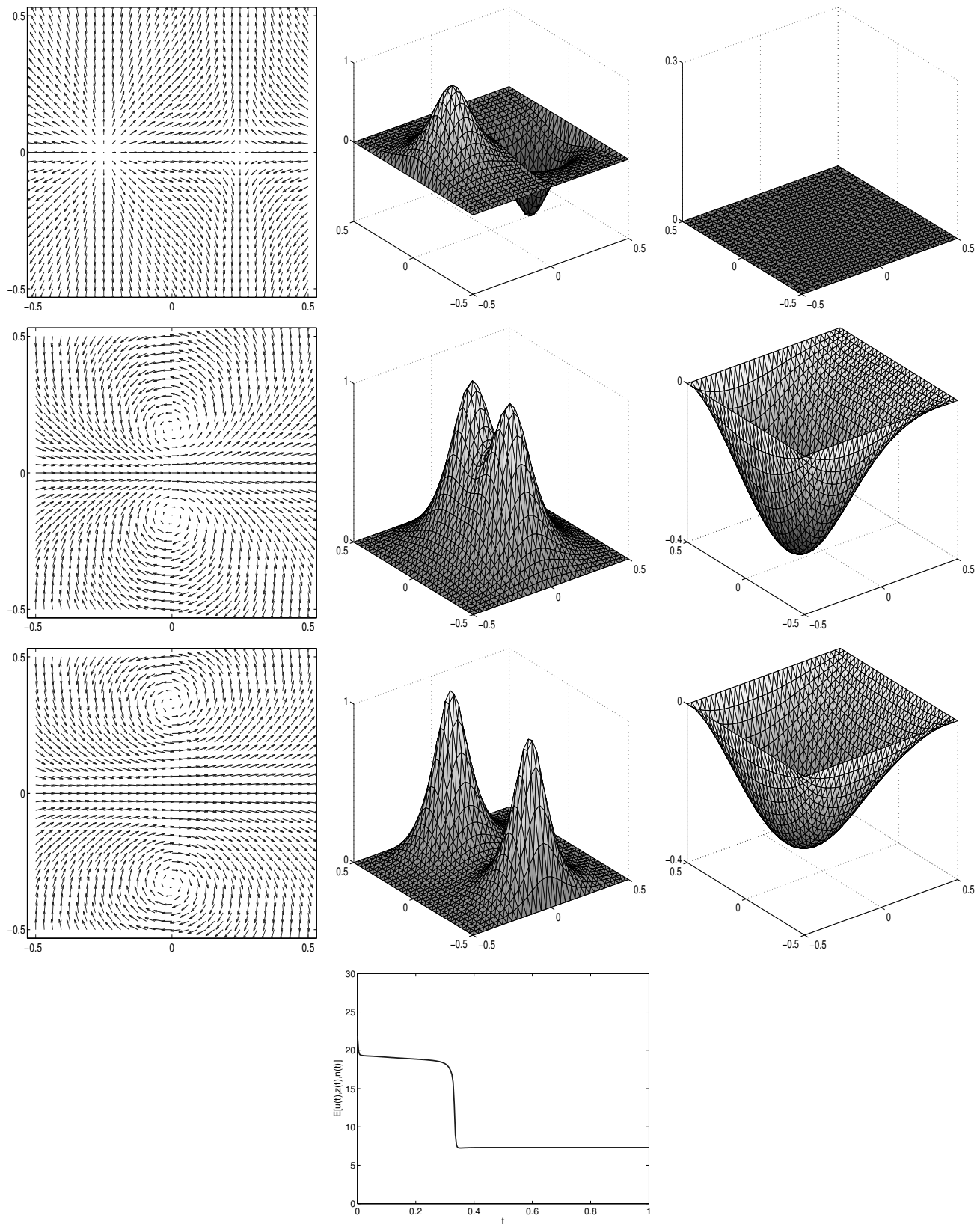

FIG. 5. Positive degree-two defect: in-plane component of the director field (left), out-of-plane component of the director field (middle), height function (right) after $j=0,48,128$ time steps. The defect splits into two rotated positive degree-one defects which do not induce local curvature, in good agreement with Section 2. The initial energy decay is followed by another strong decay when the degree-one defects are properly separated. 

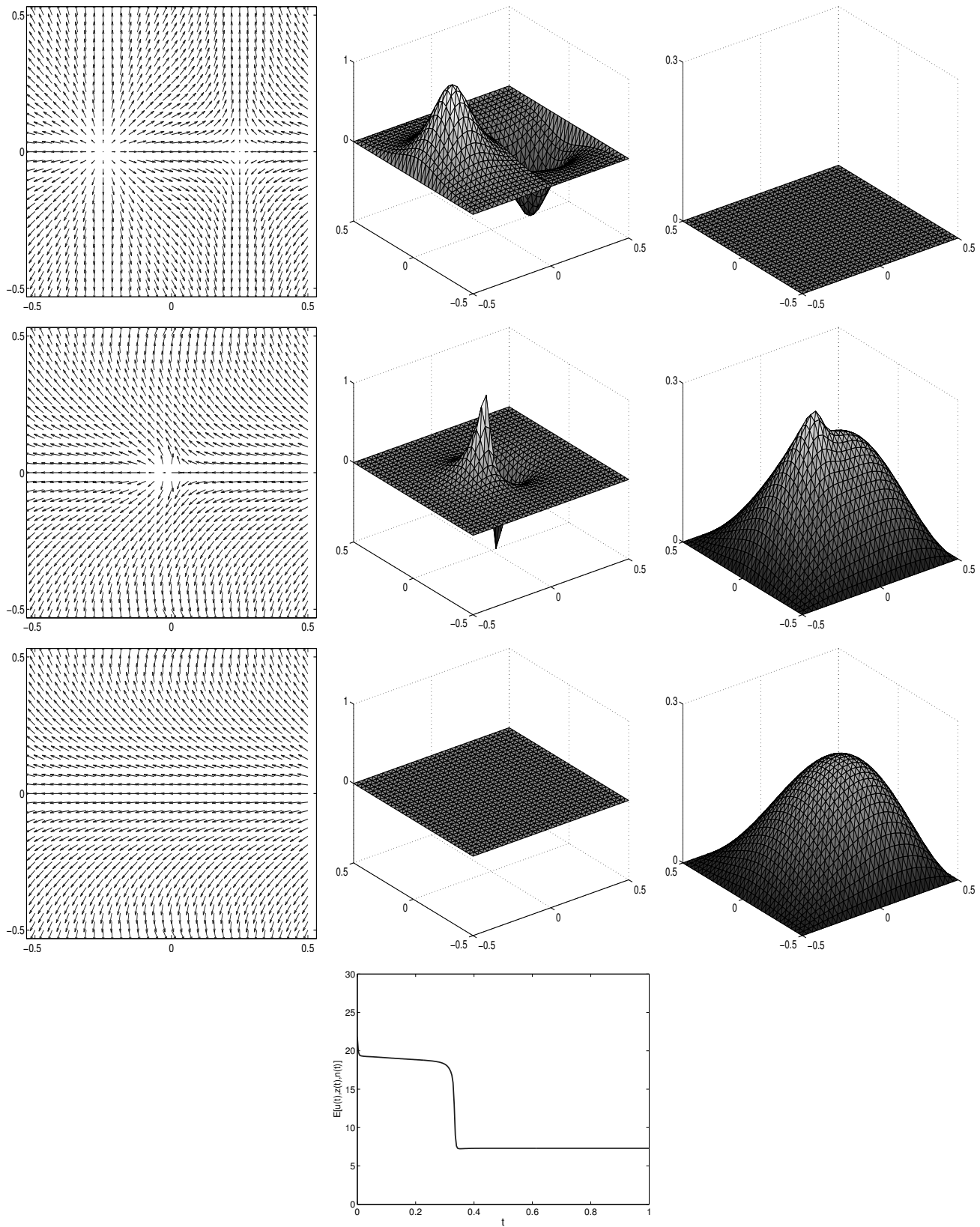

FIG. 6. Two opposite degree-one defects: in-plane component of the director field (left), out-of-plane component of the director field (middle), height function (right) after $j=0,80,96$ time steps. The surface rapidly forms a cone and a saddle-like structure which move towards each other and disappear when the attracting defects annihilate. The energy shows a strong decay when the annihilation takes place. 
The first representation is standard. To deduce the second one we make use of the orthonormal basis $\left(V_{1}, V_{2}, v\right)$, where $V_{i}=\sum_{j} g_{i j}^{(-1 / 2)} X_{j}, i=1,2$. On $\Gamma$ we have

$$
\begin{aligned}
\operatorname{div} \tilde{F}=\operatorname{tr} D \tilde{F} & =\sum_{i} V_{i}^{T} D \tilde{F} V_{i}+v^{T} D \tilde{F} v \\
& =\sum_{i, j, k} g_{i j}^{(-1 / 2)} g_{i k}^{(-1 / 2)} X_{j}^{T} D \tilde{F} X_{k}+v^{T} D \tilde{F} v \\
& =\sum_{j, k} g^{j k} \partial_{j}(F \circ X) \cdot X_{k}+v^{T} D \tilde{F} v
\end{aligned}
$$

For $F=v$ we obtain the mean curvature, i.e.,

$$
H=-\operatorname{div}_{\Gamma} v=\sum_{i, j} g^{i j} h_{i j}
$$

With this definition the unit sphere $\mathbb{S}^{2}$ has mean curvature -2 . It is now easy to check for all functions $f, f_{1}$, and $f_{2}$ the identities

$$
\left(\nabla_{\Gamma} f_{1} \cdot \nabla_{\Gamma} f_{2}\right) \circ X=\sum_{i, j} g^{i j} \partial_{i}\left(f_{1} \circ X\right) \partial_{j}\left(f_{2} \circ X\right), \quad X_{i} \cdot\left(\nabla_{\Gamma} f\right) \circ X=\partial_{i}(f \circ X) .
$$

We note that the tangential gradient $\nabla_{\Gamma} F$ of a vector field $F$ is a square matrix in $\mathbb{R}^{3 \times 3}$ whose $i$-th row is the tangential gradient of the $i$-th component of $F$. If $F$ is tangential, then it can be equivalently written as

$$
F=\sum_{k}\left(V_{k} \cdot F\right) V_{k}=\sum_{i, j, k} g_{i k}^{(-1 / 2)} g_{j k}^{(-1 / 2)} F \cdot X_{i} X_{j}=\sum_{i, j} g^{i j} F \cdot X_{i} X_{j} .
$$

Applying this expression to the tangential vector $\partial_{k} v$ yields the Weingarten equations

$$
\partial_{k} v=-\sum_{i, j} g^{i j} h_{k i} X_{j}
$$

The Laplace-Beltrami operator $\Delta_{\Gamma} f=\operatorname{div}_{\Gamma} \nabla_{\Gamma} f$ has the following expression in local coordinates

$$
\Delta_{\Gamma} f=\frac{1}{\sqrt{g}} \sum_{i, j} \partial_{i}\left(\sqrt{g} g^{i j} \partial_{j} f\right)
$$

Let $\phi$ be a smooth real-valued function on $\Gamma$ and $s \in(-\xi, \xi), \xi>0$ small enough. A normal variation of $\Gamma$ is given by the map

$$
X_{s}\left(u_{1}, u_{2}\right)=X\left(u_{1}, u_{2}\right)+s \phi\left(X\left(u_{1}, u_{2}\right)\right) v
$$

with values in a tubular neighborhood of $\Gamma$. The first variation of $E$ in (5.1) with respect to $\Gamma$ is now defined as

$$
\left\langle\delta_{\Gamma} E, \phi\right\rangle:=\left.\frac{\mathrm{d}}{\mathrm{d} s}\right|_{s=0} E\left(\Gamma_{s}, n\right) .
$$


We write $\psi^{\prime}:=\left.\partial_{s}\right|_{s=0} \psi_{s}$ for a quantity that depends on $s$, we recall the basic geometric identities

$$
\begin{array}{r}
g_{i j}^{\prime}=-2 \phi h_{i j}, \quad\left(g^{i j}\right)^{\prime}=2 \phi \sum_{k, \ell} g^{j k} g^{i \ell} h_{\ell k}, \quad v^{\prime}=-\nabla_{\Gamma} \phi, \\
\mathrm{d} \sigma^{\prime}=-\phi H \mathrm{~d} \sigma, \quad H^{\prime}=\Delta_{\Gamma} \phi+\phi\left|\nabla_{\Gamma} v\right|^{2} .
\end{array}
$$

We refer the reader to [34] for a detailed calculation and note that the first identity follows from

$$
g_{i j}^{\prime}=\left.\partial_{s}\right|_{s=0}\left(X_{i}+s \partial_{i} \phi v+s \phi \partial_{i} v\right) \cdot\left(X_{j}+s \partial_{j} \phi v+s \phi \partial_{j} v\right)=-2 \phi h_{i j} .
$$

For the side conditions we define the area and volume function as

$$
A: \Gamma \mapsto \int_{\Gamma} 1 \mathrm{~d} \sigma, \quad V: \Gamma \mapsto \frac{1}{3} \int_{\Gamma} x \cdot v \mathrm{~d} \sigma
$$

\subsection{First variation of the energy and gradient flow}

In the following we identify $n$ with its constant extension in the normal direction so that $n_{s}=n$ and therefore $n^{\prime}=0$. Notice that the variation of $E(\Gamma, n)$ with respect to $\Gamma$ does depend on the particular extension. This choice is not arbitrary: biomembranes are made of lipid bilayers and thus have a small thickness across which it is reasonable to assume no variation of the physical quantities such as $n$.

LEMMA 5.1 For a normal variation of $\Gamma$ defined by a function $\phi$ as in Section 5.1 we have

$$
\begin{aligned}
\left.\partial_{s}\right|_{s=0} \operatorname{div}_{\Gamma_{s}} n & =-\phi \nabla_{\Gamma} n: \nabla_{\Gamma} v+v^{T} \nabla_{\Gamma} n \nabla_{\Gamma} \phi, \\
\left.\partial_{s}\right|_{s=0}\left|\nabla_{\Gamma_{s}} n\right|^{2} & =-2 \phi\left(\nabla_{\Gamma} n\right)^{T}: \nabla_{\Gamma} v\left(\nabla_{\Gamma} n\right) .
\end{aligned}
$$

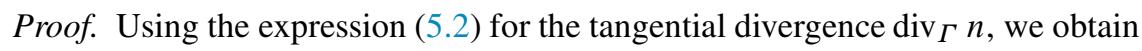

$$
\left.\partial_{s}\right|_{s=0}\left(\operatorname{div}_{\Gamma_{s}} n\right)=\left.\partial_{s}\right|_{s=0}\left(\sum_{i, j} g_{s}^{i j} \partial_{i} n \cdot X_{s, j}\right)=\sum_{i, j}\left(g^{i j}\right)^{\prime} \partial_{i} n \cdot X_{j}+g^{i j} \partial_{i} n^{\prime} \cdot X_{j}+g^{i j} \partial_{i} n \cdot X_{j}^{\prime} .
$$

Since $n^{\prime}=0$ the middle term vanishes. For the first term we use (5.6) to write

$$
\sum_{i, j}\left(g^{i j}\right)^{\prime} \partial_{i} n \cdot X_{j}=2 \phi \sum_{i, j, k, \ell} g^{j k} g^{i \ell} h_{\ell k} \partial_{i} n \cdot X_{j}=2 \phi \sum_{i, \ell} g^{i \ell} \partial_{i} n \cdot \sum_{j, k} g^{j k} h_{\ell k} X_{j} .
$$

We invoke the Weingarten equations (5.4) to identify the last factor with $-\partial_{\ell} \nu$, and (5.3) to get

$$
\sum_{i, j}\left(g^{i j}\right)^{\prime} \partial_{i} n \cdot X_{j}=-2 \phi \sum_{i, \ell} g^{i \ell} \partial_{i} n \cdot \partial_{\ell} v=-2 \phi \nabla_{\Gamma} n: \nabla_{\Gamma} v
$$

To manipulate the remaining term, we first observe that $X_{j}^{\prime}=\partial_{j} \phi v+\phi \partial_{j} v$. In view of (5.3) we see that

$$
\sum_{i, j} g^{i j} \partial_{i} n \cdot X_{j}^{\prime}=\sum_{k} v_{k} \nabla_{\Gamma} n_{k} \cdot \nabla_{\Gamma} \phi+\phi \nabla_{\Gamma} n: \nabla_{\Gamma} v
$$


Since $\sum_{k} v_{k} \nabla_{\Gamma} n_{k} \cdot \nabla_{\Gamma} \phi=v^{T} \nabla_{\Gamma} n \nabla_{\Gamma} \phi$, collecting the last two expressions leads to (5.7). To prove (5.8) we use (5.3) for $\left|\nabla_{\Gamma} n\right|^{2}$, combined with $n^{\prime}=0$, to arrive at

$$
\left.\partial_{s}\right|_{s=0}\left|\nabla_{\Gamma_{s}} n\right|^{2}=\left.\partial_{s}\right|_{s=0}\left(\sum_{i, j} g_{s}^{i j} \partial_{i} n \cdot \partial_{j} n\right)=\sum_{i, j}\left(g^{i j}\right)^{\prime} \partial_{i} n \cdot \partial_{j} n .
$$

We replace $\left(g^{i j}\right)^{\prime}$ with the expression from (5.6), and next use (5.3) to write $h_{\ell k}=-X_{k} \cdot \nabla_{\Gamma} v X_{\ell}$, whence

$$
\begin{aligned}
\left.\partial_{s}\right|_{s=0}\left|\nabla_{\Gamma_{s}} n\right|^{2} & =2 \phi \sum_{i, j, k, \ell} g^{j k} g^{i \ell} h_{\ell k} \sum_{m} \partial_{i} n_{m} \partial_{j} n_{m} \\
& =-2 \phi \sum_{m}\left(\sum_{j, k} g^{j k} \partial_{j} n_{m} X_{k}\right) \cdot \nabla_{\Gamma} v\left(\sum_{i, \ell} g^{i \ell} \partial_{i} n_{m} X_{\ell}\right) .
\end{aligned}
$$

The equivalent representation (5.2) of the surface gradient yields

$$
\left.\partial_{s}\right|_{s=0}\left|\nabla_{\Gamma_{s}} n\right|^{2}=-2 \phi \sum_{m} \nabla_{\Gamma} n_{m} \cdot \nabla_{\Gamma} v \nabla_{\Gamma} n_{m}=-2 \phi\left(\nabla_{\Gamma} n\right)^{T}: \nabla_{\Gamma} v \nabla_{\Gamma} n
$$

and completes the proof.

To compute the first variation of the energy $E(\Gamma, n)$ of $(5.1)$ we recall the differentiation rule

$$
\left.\frac{\mathrm{d}}{\mathrm{d} s}\right|_{s=0} \int_{\Gamma_{s}} \psi_{s} \mathrm{~d} \sigma_{s}=\int_{\Gamma} \psi^{\prime} \mathrm{d} \sigma-\int_{\Gamma} \psi H \phi \mathrm{d} \sigma
$$

We abreviate the first variation of the four terms in (5.1) with $I, I I, I I I$ and $I V$ and deduce for $I$

$$
\begin{aligned}
&\left.\frac{\mathrm{d}}{\mathrm{d} s}\right|_{s=0}\left(\frac{1}{2} \int_{\Gamma_{S}}(\right.\left.\left.H_{s}+\delta \operatorname{div}_{\Gamma_{s}} n\right)^{2} \mathrm{~d} \sigma_{s}\right) \\
& \quad=\int_{\Gamma}\left(H+\delta \operatorname{div}_{\Gamma} n\right)\left(H^{\prime}+\left.\delta \partial_{s}\right|_{s=0} \operatorname{div}_{\Gamma_{s}} n\right) \mathrm{d} \sigma-\frac{1}{2} \int_{\Gamma}\left(H+\delta \operatorname{div}_{\Gamma} n\right)^{2} H \phi \mathrm{d} \sigma .
\end{aligned}
$$

In light of (5.6) and (5.7), we can expand $I$ as follows:

$$
\begin{aligned}
I= & \int_{\Gamma}\left(H+\delta \operatorname{div}_{\Gamma} n\right)\left(\Delta_{\Gamma} \phi+\phi\left|\nabla_{\Gamma} v\right|^{2}-\delta \phi \nabla_{\Gamma} n: \nabla_{\Gamma} v+\delta v^{T} \nabla_{\Gamma} n \cdot \nabla_{\Gamma} \phi\right) \mathrm{d} \sigma \\
=- & \left(\nabla_{\Gamma} H, \nabla_{\Gamma} \phi\right)+\left(H\left|\nabla_{\Gamma} v\right|^{2}, \phi\right)-\delta\left(H\left(\nabla_{\Gamma} n: \nabla_{\Gamma} v\right), \phi\right)+\delta\left(H\left(v^{T} \nabla_{\Gamma} n\right), \nabla_{\Gamma} \phi\right) \\
& -\delta\left(\nabla_{\Gamma}\left(\operatorname{div}_{\Gamma} n\right), \nabla_{\Gamma} \phi\right)+\delta\left(\operatorname{div}_{\Gamma} n\left|\nabla_{\Gamma} v\right|^{2}, \phi\right)-\delta^{2}\left(\operatorname{div}_{\Gamma} n\left(\nabla_{\Gamma} n: \nabla_{\Gamma} v\right), \phi\right) \\
& +\delta^{2}\left(\operatorname{div}_{\Gamma} n\left(v^{T} \nabla_{\Gamma} n\right), \nabla_{\Gamma} \phi\right)-\frac{1}{2}\left(H\left(H+\delta \operatorname{div}_{\Gamma} n\right)^{2}, \phi\right) .
\end{aligned}
$$

For $I I$ we apply (5.8) to arrive at

$$
I I=\left.\frac{\mathrm{d}}{\mathrm{d} s}\right|_{s=0} \int_{\Gamma_{s}}\left|\nabla_{\Gamma_{s}} n\right|^{2} \mathrm{~d} \sigma=-2 \int_{\Gamma} \phi\left(\nabla_{\Gamma} n\right)^{T}: \nabla_{\Gamma} v \nabla_{\Gamma} n \mathrm{~d} \sigma-\int_{\Gamma}\left|\nabla_{\Gamma} n\right|^{2} H \phi \mathrm{d} \sigma .
$$


Then $I I$ gives no contribution because $|n|=1$ on $\Gamma$ and $n^{\prime}=0$, namely

$I I I=\left.\frac{\mathrm{d}}{\mathrm{d} s}\right|_{s=0} \int_{\Gamma_{S}}\left(\mu\left(|n|^{2}-1\right)\right) \mathrm{d} \sigma=\int_{\Gamma}\left(\mu^{\prime}\left(|n|^{2}-1\right)+2 \mu n \cdot n^{\prime}\right) \mathrm{d} \sigma-\int_{\Gamma} \mu\left(|n|^{2}-1\right) \phi H \mathrm{~d} \sigma=0$.

Finally, since (5.6) implies $(n \cdot v)^{\prime}=n \cdot v^{\prime}=-n \cdot \nabla_{\Gamma} \phi$, we can compute for $I V$

$$
I V=\left.\frac{\mathrm{d}}{\mathrm{d} s}\right|_{s=0} \int_{\Gamma_{s}} f\left(n \cdot v_{s}\right) \mathrm{d} \sigma=-\int_{\Gamma} f^{\prime}(n \cdot v) n \cdot \nabla_{\Gamma} \phi \mathrm{d} \sigma-\int_{\Gamma} f(n \cdot v) H \phi \mathrm{d} \sigma .
$$

We are now in a position to write $\delta_{\Gamma} E$. Collecting all previous expressions $I-I V$ we obtain for all $\phi \in C^{\infty}(\Gamma)$

$$
\begin{aligned}
\left\langle\frac{\delta E}{\delta \Gamma}, \phi\right\rangle= & -\left(\nabla_{\Gamma} H, \nabla_{\Gamma} \phi\right)+\left(H\left|\nabla_{\Gamma} v\right|^{2}, \phi\right)-\delta\left(H\left(\nabla_{\Gamma} n: \nabla_{\Gamma} v\right), \phi\right)+\delta\left(H\left(v^{T} \nabla_{\Gamma} n\right), \nabla_{\Gamma} \phi\right) \\
& -\delta\left(\nabla_{\Gamma}\left(\operatorname{div}_{\Gamma} n\right), \nabla_{\Gamma} \phi\right)+\delta\left(\operatorname{div}_{\Gamma} n\left|\nabla_{\Gamma} v\right|^{2}, \phi\right)-\delta^{2}\left(\operatorname{div}_{\Gamma} n\left(\nabla_{\Gamma} n: \nabla_{\Gamma} v\right), \phi\right) \\
& +\delta^{2}\left(\operatorname{div}_{\Gamma} n\left(v^{T} \nabla_{\Gamma} n\right), \nabla_{\Gamma} \phi\right)-\frac{1}{2}\left(H\left(H+\delta \operatorname{div}_{\Gamma} n\right)^{2}, \phi\right) \\
& -\lambda\left(\left(\nabla_{\Gamma} n\right)^{T}: D v\left(\nabla_{\Gamma} n\right), \phi\right)-\frac{\lambda}{2}\left(H\left|\nabla_{\Gamma} n\right|^{2}, \phi\right)-\frac{1}{2 \varepsilon^{2}}\left(f^{\prime}(n \cdot v) n, \nabla_{\Gamma} \phi\right) \\
& -\frac{1}{2 \varepsilon^{2}}(H f(n \cdot v), \phi),
\end{aligned}
$$

The variation with respect to $n$ is given by

$$
\left\langle\frac{\delta E}{\delta n}, m\right\rangle=\delta\left(H+\delta \operatorname{div}_{\Gamma} n, \operatorname{div}_{\Gamma} m\right)+\lambda\left(\nabla_{\Gamma} n, \nabla_{\Gamma} m\right)+(2 \mu n, m)+\frac{1}{2 \varepsilon^{2}}\left(f^{\prime}(n \cdot v), m \cdot v\right),
$$

for all $m \in C^{\infty}\left(\Gamma ; \mathbb{R}^{3}\right)$. This expression simplifies if we impose tangential variations $m \in$ $C^{\infty}\left(\Gamma ; T_{n} \mathbb{S}^{2}\right):=\left\{v \in C^{\infty}\left(\Gamma ; \mathbb{R}^{3}\right): v(x) \in T_{n(x)} \mathbb{S}^{2}\right.$ a.e. $\left.x \in \Gamma\right\}$ so that $(\mu n, m)=0$.

We simulate the evolution of $\Gamma$ and $n$ via a relaxation dynamics, which is an $L^{2}$-gradient flow. If $v$ denotes the normal velocity of $\Gamma$, we then have to solve the following system of PDE on $\Gamma$

$$
\begin{aligned}
\langle v, \phi\rangle & =-\left\langle\frac{\delta E}{\delta \Gamma}, \phi\right\rangle \quad \text { for all } \phi \in C^{\infty}(\Gamma), \\
\left\langle\partial_{t} n, m\right\rangle & =-\left\langle\frac{\delta E}{\delta n}, m\right\rangle \quad \text { for all } m \in C^{\infty}\left(\Gamma ; T_{n} \mathbb{S}^{2}\right),
\end{aligned}
$$

subject to the constraint that $n(t, x) \in \mathbb{S}^{2}$ for almost every $(t, x)$.

Remark 5.1. Let $\Phi:[0, T) \times \mathbb{R}^{3} \rightarrow \mathbb{R}^{3}$ be the flow of the evolution of $\Gamma(t)$ for $t \in[0, T)$, that is, $\left.\Phi(0, \cdot)\right|_{\Gamma(0)}=\left.\mathrm{id}\right|_{\Gamma(0)}$ and $\partial_{t} \Phi(t, x)=v(t, \Phi(t, x)) v(t, \Phi(t, x))$. Then

$$
\left.\frac{\mathrm{d}}{\mathrm{d} s}\right|_{s=t} E(\Gamma(s), n(s, \Phi(s, \cdot)))=\left\langle\frac{\partial E}{\partial \Gamma}, v\right\rangle+\left\langle\frac{\partial E}{\partial n},\left.\frac{\mathrm{d}}{\mathrm{d} s}\right|_{s=t} n(s, \Phi(s, \cdot))\right\rangle .
$$

Since $n$ is constant in normal direction we have that

$$
\begin{aligned}
\left.\frac{\mathrm{d}}{\mathrm{d} s}\right|_{s=t} n(s, \Phi(s, x)) & =\partial_{t} n(t, \Phi(t, x))+\nabla n(t, \Phi(t, x)) \cdot v(t, \Phi(t, x)) v(t, \Phi(t, x)) \\
& =\partial_{t} n(t, \Phi(t, x)) .
\end{aligned}
$$


Although, we do not prove a discrete energy law for the $L^{2}$ gradient flow in the closed surface case we have that

$$
\left.\frac{\mathrm{d}}{\mathrm{d} s}\right|_{s=t} E(\Gamma(s), n(s, y(s)))=-\langle v, v\rangle-\left\langle\partial_{t} n, \partial_{t} n\right\rangle \leqslant 0,
$$

and expect a discrete energy reduction in the numerical experiments.

\subsection{Finite elements on surfaces}

The time discretization of the gradient flow leads to a family of surfaces $\left(\Gamma^{j}\right)_{j \in \mathbb{N}}$ related to the time-steps $t_{j}$. Let $\Gamma_{h}^{j}$ be a polyhedral approximation of $\Gamma^{j}$ consisting of flat triangles with maximal diameter less than $h>0$. Since $\Gamma_{h}^{j}$ is the union of triangles $T \in \mathcal{J}^{j}$, we identify the triangulation $\mathcal{J}^{j}$ and the discrete surface $\Gamma_{h}^{j}$. Let $\mathbb{V}^{j}=\mathbb{V}\left(\Gamma_{h}^{j}\right)$ be the space of all continuous functions on $\Gamma_{h}^{j}$ whose restriction to the triangles are affine. Moreover let $\mathbb{C}_{0}^{j}=\mathbb{C}_{0}\left(\Gamma_{h}^{j}\right)$ denote the space of all functions that are constant on every triangle and define the averaging operator $Q^{j}: \mathbb{C}_{0}^{j} \rightarrow \mathbb{V}^{j}$, $v \mapsto \sum_{a} v_{a} \varphi_{a}$, where $v_{a}:=\frac{1}{\left|\omega_{a}\right|} \int_{\omega_{a}} v(x) \mathrm{d} x$ and $\left(\varphi_{a}\right)_{a \in \mathfrak{n}^{j}}$ is the standard nodal basis of $\mathbb{V}^{j}$. Here $\eta^{j}=\eta\left(t_{j}\right)=\left\{a_{1}\left(t_{j}\right), \ldots, a_{N}\left(t_{j}\right)\right\}$ is the set of all nodes in $\Gamma_{h}^{j}, \omega_{a}=\operatorname{supp} \varphi_{a}$, and the map $t_{j} \mapsto a\left(t_{j}\right) \in \mathbb{R}^{3}$ is the trajectory on which a node $a \in \mathfrak{n}$ moves in time as the surface is changing its shape. If $\widetilde{v}^{j} \in \mathbb{C}_{0}^{j}$ stands for the the piecewise constant outer normal to $\Gamma_{h}^{j}$, then

$$
v^{j}:=Q^{j}\left(\widetilde{v}^{j}\right) \in\left[\mathbb{V}^{j}\right]^{3}
$$

is a piecewise linear reconstruction of $\widetilde{v}^{j}$. For a given function $\phi^{j-1} \in \mathbb{V}^{j-1}$ we define $G^{j} \circ \phi^{j-1} \in$ $\mathbb{V}^{j}$ by $\left(G^{j} \circ \phi^{j-1}\right)\left(a\left(t_{j}\right)\right)=\phi^{j-1}\left(a\left(t_{j-1}\right)\right)$ for all $a \in \eta^{j}$. For a better readability we will denote $G^{j} \circ \phi^{j-1}$ also by $\phi^{j-1}$ if no confusion is possible.

\subsection{Discretization}

We start with the time discretization. Given the surface $\Gamma^{j-1} \subset \mathbb{R}^{3}$ at time $t_{j-1}$ we follow the ideas in [14] to parametrize $\Gamma^{j}$ at time $t_{j}$ over $\Gamma^{j-1}$. We thus look for $X^{j}: \Gamma^{j-1} \rightarrow \mathbb{R}^{3}$ and set $\Gamma^{j}=X^{j}\left(\Gamma^{j-1}\right)$. As in [3], we approximate the normal velocity via

$$
v^{j} \approx \frac{1}{\tau}\left(X^{j}-\operatorname{id}_{\Gamma^{j-1}}\right) \cdot v^{j-1},
$$

where $v^{j-1}: \Gamma^{j-1} \rightarrow \mathbb{S}^{2}$ is the outer unit normal to $\Gamma^{j-1}$. Now, again, using an idea from [14] to compute $H^{j}$ we discretize the crucial geometric identity $\Delta_{\Gamma} X=H v[14,15]$ :

$$
\Delta_{\Gamma^{j-1}} X^{j}=H^{j} v^{j-1} .
$$

Notice that we compute the scalar mean curvature $H^{j}$, as in [3], and not the mean curvature vector, as in $[2,9,15]$. For the evolution of $n$ we use the techniques from [5].

In order to formulate the fully discrete evolution concisely we set $\operatorname{Div}_{\Gamma_{h}^{j-1}} n^{j-1}:=$ $Q^{j}\left(\operatorname{div}_{\Gamma_{h}^{j-1}} n^{j-1}\right)$, denote by $(\cdot, \cdot)_{\Gamma_{h}^{j-1}}$ the standard $L^{2}$-inner product on $\Gamma_{h}^{j-1}$, and we define 
$\Psi_{\delta_{\Gamma} E}^{j-1, j}=\Psi_{\delta_{\Gamma} E}^{j-1, j}\left(H^{j}, H^{j-1}, v^{j-1}, n^{j-1}\right) \in \mathbb{V}^{j-1}$ to be the representation of a semi-implicit discretization of $\frac{\delta E}{\delta \Gamma}$ given by

$$
\begin{aligned}
& \left(\Psi_{\delta_{\Gamma} E}^{j-1, j}, \phi\right)=-\left(\nabla_{\Gamma_{h}^{j-1}} H^{j}, \nabla_{\Gamma_{h}^{j-1}} \phi\right)_{\Gamma_{h}^{j-1}}+\left(H^{j-1}\left|\nabla_{\Gamma_{h}^{j-1}} v^{j-1}\right|^{2}, \phi\right)_{\Gamma_{h}^{j-1}} \\
& -\delta\left(H^{j}\left(\nabla_{\Gamma_{h}^{j-1}} n^{j-1}: \nabla_{\Gamma_{h}^{j-1}} v^{j-1}\right), \phi\right)_{\Gamma_{h}^{j-1}}+\delta\left(H^{j}\left(\left(v^{j-1}\right)^{T} \nabla_{\Gamma_{h}^{j-1} n^{j-1}}\right), \nabla_{\Gamma_{h}^{j-1}} \phi\right)_{\Gamma_{h}^{j-1}} \\
& -\delta\left(\nabla_{\Gamma_{h}^{j-1}}\left(\operatorname{Div}_{\Gamma_{h}^{j-1} n^{j-1}}\right), \nabla_{\Gamma_{h}^{j-1}} \phi\right)_{\Gamma_{h}^{j-1}}+\delta\left(\operatorname{Div}_{\Gamma_{h}^{j-1} n^{j-1}}\left|\nabla_{\Gamma_{h}^{j-1}} v^{j-1}\right|^{2}, \phi\right)_{\Gamma_{h}^{j-1}} \\
& -\delta^{2}\left(\operatorname{Div}_{\Gamma_{h}^{j-1}} n^{j-1}\left(\nabla_{\Gamma_{h}^{j-1}} n^{j-1}: \nabla_{\Gamma_{h}^{j-1}} v^{j-1}\right), \phi\right)_{\Gamma_{h}^{j-1}} \\
& +\delta^{2}\left(\operatorname{Div}_{\Gamma_{h}^{j-1}} n^{j-1}\left(\left(v^{j-1}\right)^{T} \nabla_{\Gamma_{h}^{j-1}} n^{j-1}\right), \nabla_{\Gamma_{h}^{j-1}} \phi\right)_{\Gamma_{h}^{j-1}} \\
& -\frac{1}{2}\left(H^{j}\left(H^{j-1}+\delta \operatorname{Div}_{\Gamma_{h}^{j-1}} n^{j-1}\right)^{2}, \phi\right)_{\Gamma_{h}^{j-1}} \\
& -\lambda\left(\left(\nabla_{\Gamma_{h}^{j-1}} n^{j-1}\right)^{T}: \nabla_{\Gamma_{h}^{j-1}} v^{j-1}\left(\nabla_{\Gamma_{h}^{j-1}} n^{j-1}\right), \phi\right)_{\Gamma_{h}^{j-1}}-\frac{\lambda}{2}\left(H^{j}\left|\nabla_{\Gamma_{h}^{j-1}} n^{j-1}\right|^{2}, \phi\right)_{\Gamma_{h}^{j-1}} \\
& -\frac{1}{2 \varepsilon^{2}}\left(f^{\prime}\left(n^{j-1} \cdot v^{j-1}\right) n^{j-1}, \nabla_{\Gamma_{h}^{j-1}} \phi\right)_{\Gamma_{h}^{j-1}}-\frac{1}{2 \varepsilon^{2}}\left(H^{j} f\left(n^{j-1} \cdot v^{j-1}\right), \phi\right)_{\Gamma_{h}^{j-1}},
\end{aligned}
$$

for all $\phi \in \mathbb{V}^{j-1}$.

\subsection{Volume and mass constraints}

We now recall a method for the conservation of area and volume proposed by Bonito, Nochetto, and Pauletti in [9]. We introduce the extended energy

$$
F(\Gamma, n)=E(\Gamma, n)+\rho_{1}\left(V(\Gamma)-V\left(\Gamma^{0}\right)\right)+\rho_{2}\left(A(\Gamma)-A\left(\Gamma^{0}\right)\right),
$$

and compute the first variation with respect to $\Gamma$ :

$$
\left\langle\frac{\delta F}{\delta \Gamma}, \phi\right\rangle=\left\langle\frac{\delta E}{\delta \Gamma}, \phi\right\rangle+\rho_{1} \int_{\Gamma} \phi \mathrm{d} \sigma-\rho_{2} \int_{\Gamma} H \phi \mathrm{d} \sigma .
$$

Following [9] we compute in each time-step the velocities $v_{E}^{j}, v_{V}^{j}, v_{A}^{j}$ via

$$
\left(v_{E}^{j}, \phi\right)_{\Gamma_{h}^{j-1}}=-\left\langle\Psi_{\delta_{\Gamma} E}^{j-1, j}, \phi\right\rangle, \quad\left(v_{V}^{j}, \phi\right)_{\Gamma_{h}^{j-1}}=-(1, \phi)_{\Gamma_{h}^{j-1}}, \quad\left(v_{A}^{j}, \phi\right)_{\Gamma_{h}^{j-1}}=\left(H^{j}, \phi\right)_{\Gamma_{h}^{j-1}},
$$

and define the function

$$
f^{j}: \mathbb{R}^{2} \rightarrow \mathbb{R}^{2}, \quad\left(\rho_{1}, \rho_{2}\right) \mapsto\left[\begin{array}{l}
V\left(\Gamma^{j}\left(\rho_{1}, \rho_{2}\right)\right)-V\left(\Gamma^{j-1}\right) \\
A\left(\Gamma^{j}\left(\rho_{1}, \rho_{2}\right)\right)-A\left(\Gamma^{j-1}\right)
\end{array}\right],
$$

for $\Gamma^{j}\left(\rho_{1}, \rho_{2}\right)=X\left(\Gamma^{j-1}\right), X=X^{j-1}+\tau\left(v_{E}^{j}+\rho_{1} v_{V}^{j}+\rho_{2} v_{A}^{j}\right)$. Now we use a Newton iteration to compute a solution $\left(\rho_{1}^{j}, \rho_{2}^{j}\right)$ of $f\left(\rho_{1}, \rho_{2}\right)=0$ and set $X^{j}=X^{j-1}+\tau\left(v_{E}^{j}+\rho_{1}^{j} v_{V}^{j}+\rho_{2}^{j} v_{A}^{j}\right)$ and $\Gamma^{j}=X^{j}\left(\Gamma^{j-1}\right)$. 
5.6 Semi-implicit fully discrete gradient flow with constraints

We start with an initial polyhedral surface $\Gamma_{h}^{0}$, time-step size $\tau>0$, parameters $\varepsilon, \delta$, $\lambda$, and an initial director field $n^{0} \in\left[\mathbb{V}^{0}\right]^{3}$ with $\left|n^{0}(a)\right|=1$ for all $a \in \eta_{h}^{0}$. We set $j:=1$ and iterate on $j$ the following steps:

(1) Compute $\left(\widetilde{X}^{j}, H^{j}\right) \in\left[\mathbb{V}^{j-1}\right]^{3} \times \mathbb{V}^{j-1}$ satisfying

$$
\begin{aligned}
\frac{1}{\tau}\left(\left(\widetilde{X}^{j}-X^{j-1}\right) \cdot v^{j-1}, \phi\right)_{\Gamma_{h}^{j-1}} & =-\left(\Psi_{\delta_{\Gamma} E}^{j-1, j}, \phi\right)_{\Gamma_{h}^{j-1}}, \\
\left(\nabla_{\Gamma_{h}^{j-1}} \widetilde{X}^{j}, \nabla_{\Gamma_{h}^{j-1}} \eta\right)_{\Gamma_{h}^{j-1}} & =-\left(H^{j}, \eta \cdot v^{j-1}\right)_{\Gamma_{h}^{j-1}},
\end{aligned}
$$

for all $\phi \in \mathbb{V}^{j-1}$ and all $\eta \in \mathbb{V}\left(\Gamma_{h}^{j-1} ; \mathbb{R}^{3}\right)$.

(2) Set

$$
v_{E}=\frac{1}{\tau}\left(\widetilde{X}^{j}-X^{j-1}\right) \cdot v^{j-1}, \quad v_{V}=-1, \quad v_{A}=H^{j},
$$

and compute $\left(\rho_{1}^{j}, \rho_{2}^{j}\right)$ such that $f^{j}\left(\rho_{1}^{j}, \rho_{2}^{j}\right)=0$. Set

$$
\begin{gathered}
X^{j}=X^{j-1}+\tau\left(v_{E}+\rho_{1}^{j} v_{V}+\rho_{2}^{j} v_{A}\right) v^{j-1}, \quad \Gamma_{h}^{j}=\left\{X^{j}(x): x \in \Gamma_{h}^{j-1}\right\}, \\
n^{j-1}:=G^{j} \circ n^{j-1} .
\end{gathered}
$$

(3) Compute $\widetilde{\mathrm{d}}_{t} n^{j} \in \mathbb{F}\left[n^{j-1}\right]$ with

$$
\begin{aligned}
\left(\widetilde{\mathrm{d}}_{t} n^{j}, m\right)_{\Gamma_{h}^{j}}+\tau \lambda\left(\nabla_{\Gamma_{h}^{j}} \widetilde{\mathrm{d}}_{t} n^{j}, \nabla_{\Gamma_{h}^{j}} m\right)_{\Gamma_{h}^{j}} & \\
=-\lambda\left(\nabla_{\Gamma_{h}^{j}} n^{j-1}, \nabla_{\Gamma_{h}^{j}} m\right)_{\Gamma_{h}^{j}}-\delta\left(H^{j}+\delta \operatorname{Div}_{\Gamma_{h}^{j-1}} n^{j-1}, \operatorname{div}_{\Gamma_{h}^{j}} m\right)_{\Gamma_{h}^{j}} & -\frac{1}{2 \varepsilon^{2}}\left(f^{\prime}\left(n^{j-1} \cdot v^{j}\right), m \cdot v^{j}\right)_{\Gamma_{h}^{j}},
\end{aligned}
$$

for all $m \in \mathbb{F}\left[n^{j-1}\right]$, where the latter is the space of vector-valued continuous piecewise linear functions that are orthogonal to $n^{j-1}$ at the nodes $\eta^{j-1}$.

(4) For all $a \in \eta^{j}$ set

$$
n^{j}(a)=\frac{n^{j-1}(a)+\tau \widetilde{\mathrm{d}}_{t} n^{j}(a)}{\left|n^{j-1}(a)+\tau \widetilde{\mathrm{d}}_{t} n^{j}(a)\right|} .
$$

(5) Set $X^{j}:=G^{j} \circ X^{j}=\operatorname{id}_{\Gamma_{h}^{j}}, j=j+1$ and go to (1).

Remarks 5.1. (i) Setting $\left(\rho_{1}^{j}, \rho_{2}^{j}\right)=0$ in 2 reduces the iteration to an $L^{2}$-flow for $E(\Gamma, n)$.

(ii) Solvability of the system in Step (1) can be established by arguing as in [3]; see also [2].

\section{Numerical experiments for the nonlinear model}

In our numerical experiments for the model on closed surfaces we distinguish the cases $\varepsilon=\infty$ and $\varepsilon \ll 1$, where $\varepsilon=\infty$ means that the term penalizing variations of $n$ from a prescribed angle relative to the surface normal, i.e., the term including $f$ in $E(\Gamma, n)$, is omitted. The realization of 
the volume and area constraints via the Newton iteration outlined above allowed us to satisfy the conservation of these quantities up to machine precision. As a stopping criterion for the discrete evolutions we used that the change of the discrete energy

$$
E_{h}\left(\Gamma_{h}, n_{h}\right):=\frac{1}{2} \int_{\Gamma_{h}}\left(H+\delta Q\left(\operatorname{div}_{\Gamma_{h}} n_{h}\right)\right)^{2} \mathrm{~d} \sigma+\frac{\lambda}{2} \int_{\Gamma_{h}}\left|\nabla_{\Gamma_{h}} n_{h}\right|^{2} \mathrm{~d} \sigma+\frac{1}{2 \varepsilon^{2}} \int_{\Gamma_{h}} f\left(n_{h} \cdot v\right) \mathrm{d} \sigma
$$

in two consecutive time-steps was less than $10^{-5}$. For significantly smaller stopping criteria we observed in some of our experiments that the evolution became unstable which is related to unfavorable tangential motions on the surface which eventually lead to singularities in the mesh. Given that there is no stability analysis for the closed surface case an optimal time-step size can not be computed and in our simulations we mainly use $\tau=h^{4}$. Moreover we work only on two different meshes so there is no evidence for assuming a scaling or special asymptotics for $\tau$. We believe that by employing mesh regularization techniques such as those in [2, 9] we could use larger time steps and a smaller stopping criterion. The evolution equation for the director field $n$ on $\Gamma$ is a second order parabolic equation and should therefore also work for $\tau \sim h^{2}$ as was shown in [16]. Our proposed algorithm was implemented in Matlab and all experiments were carried out on a standard desktop (Intel Core (TM) 2 Quad CPU Q6600 @ 2.40 GHz). The CPU-time needed for the calculation of one step with 2048 elements including the assembly of the system matrices was around 0.05 seconds. In all figures displayed below the color scale was chosen so that low values of a quantity are represented by dark and large values by bright colors. All displayed arrows have unit length and are scaled for graphical purposes.

\subsection{Surfactants}

To simulate surfactants we omit the penalty term which corresponds to the choice $\varepsilon=\infty$.

6.1.1 Perturbed sphere with volume constraint. We set $\delta=1, \lambda=5, \tau=h^{4}$, choose as initial surface $\Gamma_{h}^{0}$ a perturbation of the unit sphere, and as initial director field $n^{0}$ a perturbation of the discrete outer unit normal $v^{0}$. The perturbations were realized by displacing the nodes of a triangulation $\mathcal{J}$ of the unit sphere with 2048 elements in normal direction and the unit normals by random vectors with magnitudes bounded by 0.1 and 0.05 , respectively. The first summand of the continuous energy functional $E(\Gamma, n)$ vanishes for $n=v$ and this director field is stationary for the Dirichlet energy subject to a unit-length constraint, i.e., the outer unit normal of the sphere is a harmonic map into the unit sphere. Therefore, we expect that the pair $\left(\mathbb{S}^{2}, v\right)$ is a stationary point for $E(\Gamma, n)$ subject to a volume constraint. The snapshots of the discrete evolution shown in Figure 7 confirm this expected behavior and the monotone energy decay displayed in the right plot of Figure 7 suggests that the chosen discretization parameters are sufficiently small to compute a stable and accurate approximation of the exact evolution. When we stopped our calculations the discrete energy was $E_{h}=62.8516$, which is an accurate approximation of the value $E\left(\mathbb{S}^{2}, v\right)=20 \pi$, i.e., the absolute error is $\left|E_{h}-E\left(\mathbb{S}^{2}, v\right)\right|<0.02$. The alignment of the director field $n$ describing the orientation of the surfactant molecules and the surface normal is visualized by the coloring of the displayed arrows and is an effect which is frequently observed for surfactants. We remark that our simulations showed that for larger values of $\lambda$ we could use larger time steps.

6.1.2 4-4-1 Ellipsoid with area and volume constraint. We set $\delta=1, \lambda=1$, and $\tau=h^{3}$. To define the initial surface $\Gamma^{0}$ we employ a triangulation $\mathcal{S}$ of the unit sphere with 768 elements and 

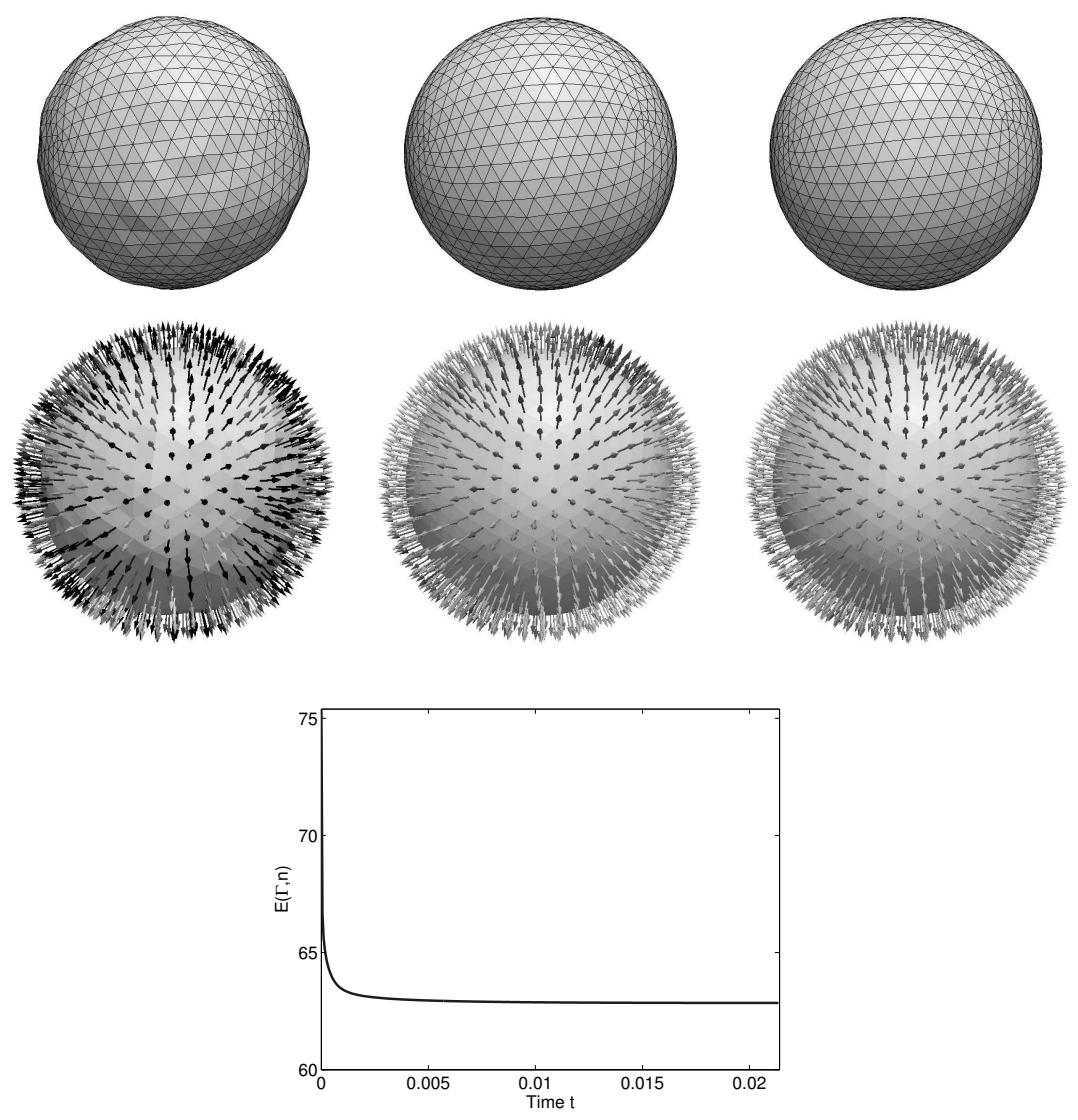

FIG. 7. Evolution from a perturbed sphere in the surfactant case with volume constraint: snapshots of the evolution after $j=1,50$ and 400 time-steps. The arrows are colored by $n \cdot v$. The surface normal and the director field align and the surface attains a stable state that coincides with a sphere. The plot depicts the decay of the energy during the evolution.

deform the triangulated sphere by mapping its nodes contained in $\eta$ onto a 4-4-1 ellipsoid, i.e., we set

$$
\eta^{0}=\left\{\widetilde{a}: \widetilde{a}=4 a_{1} e_{1}+4 a_{2} e_{2}+e_{3} \text { for } a=\left(a_{1}, a_{2}, a_{3}\right) \in \eta\right\},
$$

and this defines a triangulation $\Gamma_{h}^{0}$ of the ellipsoid. The initial director field was defined by setting $n^{0}=v^{0}$. Incorporating volume and area constraints in the evolution allows us to compare the qualitative behavior of our model with well known observations for the Helfrich flow which is included in our model and corresponds to the uncoupled flow defined through $\delta=0$, i.e., without spontaneous curvature. The upper row in Figure 8 displays a cut through the discrete surfaces at different times within the evolution for $\delta=1$. We observe that the surface develops the shape of a discocyte. Qualitatively, such shapes have been observed to be stationary for the Helfrich flow and we plot in the second row of Figure 8 the discrete surfaces for this model, i.e., for our scheme with $\delta=0$. We observe that the coupling with the director field leads to a deceleration and the shape of the discocyte is not as pronounced as in the uncoupled case. The plots including the director field 


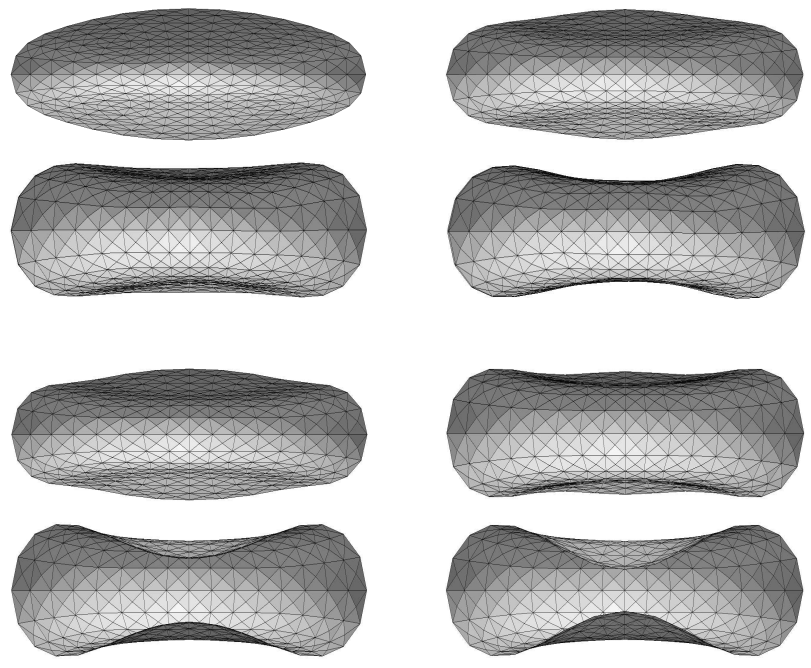

FIG. 8. Evolution from a prolate 4-4-1 ellipsoid in the surfactant case with volume and area constraint: snapshots of the evolution after $j=50,200,600$ and 1200 time-steps. The upper plots show the evolution in the presence of surfactants $(\delta=1)$ while the third and fourth row shows the Helfrich-flow $(\delta=0)$. The coupling of the surface and the director field decelerates the evolution and leads to a less pronounced shape.

in Figure 9 of the nearly stationary configuration in the coupled case show that the director field is aligned with the surface normal in regions where the surface can be approximated by a sphere, i.e., in regions where the unit normal is a harmonic map.

\subsection{Biomembranes}

In our second set of experiments for the full model on closed surfaces we use $\varepsilon=1 / \sqrt{20}$ and consider initial director fields on the sphere with different topological properties. In the gel phase the director field prefers to have a fixed angle with respect to the normal to the surface. As in the flat case we restrict ourselves to $\pi / 2$, which corresponds to $\xi_{0}=0$. Throughout the first subsection the underlying triangulation of the unit sphere consists of 8192 elements, we always choose $\tau=h^{4}$, $\lambda=1$, and we enforce conservation of the enclosed volume. For the last experiment we use a finer triangulation, consisting of 12288 elements, while $\tau$ and $\lambda$ remain unchanged. To magnify relevant effects of the coupling between the director field and the curvature of the surface we employ different values of $\delta$.

6.2.1 Positive degree-one defects. Given a point $a=\left(a_{1}, a_{2}, a_{3}\right)$ on the sphere we let $(r, \theta)$ be the polar coordinates of the first two components of $a$, i.e., $\left(a_{1}, a_{2}\right)=r(\cos \theta, \sin \theta)$. Since deviations of $n \cdot v$ from 0 are penalized by a Ginzburg Landau term we use the characteristic profile of tanh to regularize the initial singularities. Thus, we set $\varphi_{\varepsilon}(r)=\tanh (r / \varepsilon)$ and use this function in the extension of a director field in the neighborhood of a singularity. Then the transition of $n \cdot v$ from 0 to 1 is on an annulus of width $\varepsilon$ around the center of the singularity where the value 1 is taken. Note that $\varepsilon$ has to be chosen big enough so that the transition can be resolved properly by the mesh. We define three initial director fields $n_{0}$ that have defects of positive degree-one at the north 

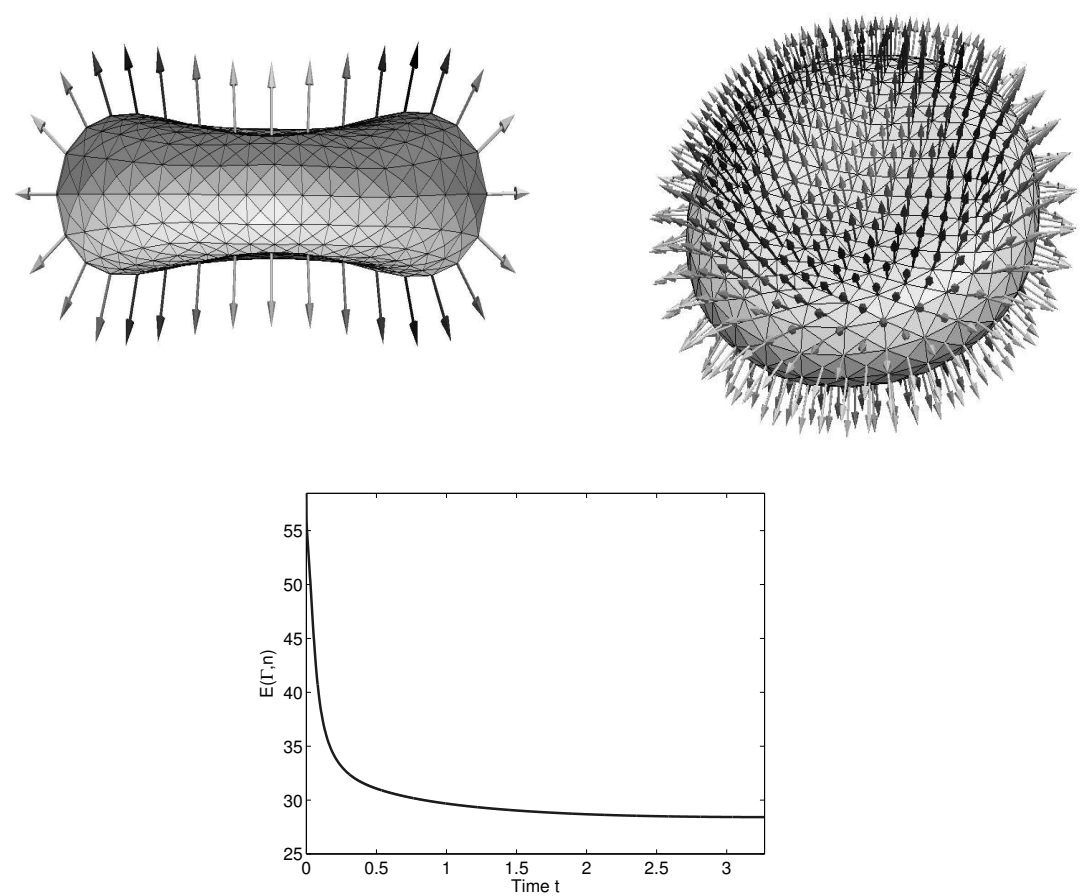

FIG. 9. Evolution from a prolate 4-4-1 ellipsoid in the surfactant case with volume and area constraint: snapshots of the final state of the evolution in the presence of surfactants $(\delta=1)$. The director field is colored by the deviation of $n \cdot v$ from 1 and we observe that in regions where the surface is approximated by a sphere the director aligns with the surface normal. A monotone decay of the energy can be observed in the right plot.

and south pole as follows.

(i) Two outward pointing defects:

$$
n^{0}(a)=\left(\varphi_{\varepsilon}(r) \cos (\theta), \varphi_{\varepsilon}(r) \sin (\theta), \operatorname{sign}\left(a_{3}\right)\left(1-\varphi_{\varepsilon}(r)^{2}\right)^{1 / 2}\right) .
$$

(ii) Two $90^{\circ}$-rotated defects:

$$
n^{0}(a)=\left(\varphi_{\varepsilon}(r) \cos (\theta+\pi / 2), \varphi_{\varepsilon}(r) \sin (\theta+\pi / 2), \operatorname{sign}\left(a_{3}\right)\left(1-\varphi_{\varepsilon}(r)^{2}\right)^{1 / 2}\right) .
$$

(iii) Inward and outward pointing defect:

$$
n^{0}(a)=\frac{(0,0,1)-\varphi_{\varepsilon}(r) a_{3} a}{\left|(0,0,1)-\varphi_{\varepsilon}(r) a_{3} a\right|} .
$$

The initial director fields are shown from different perspectives in the rows of Figure 10 for (i)-(iii), respectively. We use $\delta=0.5, \delta=1$, and $\delta=0.75$ for the settings defined by (i), (ii), and (iii), respectively, in order to enhance the effect of the different defects on the shape of the surface.

Snapshots of the surface and the director field during the discrete evolutions defined with the initial data from (i) and (iii) are shown in Figure 11 and 12, respectively. The observed results are in 
very good agreement with our theoretical predictions from Section 2: The surface develops outward cones of negative curvature at defects for which the tangential part of the director field points away from the defect and inward cones if the director field points towards the defect. These configurations show certain analogies with stomatocyte and echinocyte shapes observed in experiments, cf., e.g., [24]. For the initial data defined in (i) and the corresponding snapshots displayed in Figure 11 we see that the surface also develops large curvature in a neighborhood of the equator. This is related to the fact that the director field is purely normal along this line thereby leading to a large contribution from the penalty term which induces local curvature. In the plots shown in Figure 12, where the initial director field from (iii) is tangential along the equator, the surface does not develop such effects. For the initial data defined in (ii) we did not observe changes of the initial surface which again matches our earlier observation that a $90^{\circ}$-rotated defect of positive degree one is divergence free and hence does not enforce local curvature. We finally remark that the qualitative behavior of the surfaces with initial director fields defined in (i)-(iii) was nearly independent of the choice of the preferred angle $\xi_{0}$. This justifies the earlier discussed simplification of the previous sections to consider only the tangential part of the director field and to analyze its influence on the local shape of the surfaces. In Figure 13 we see the monotone decay of energy during the evolutions.
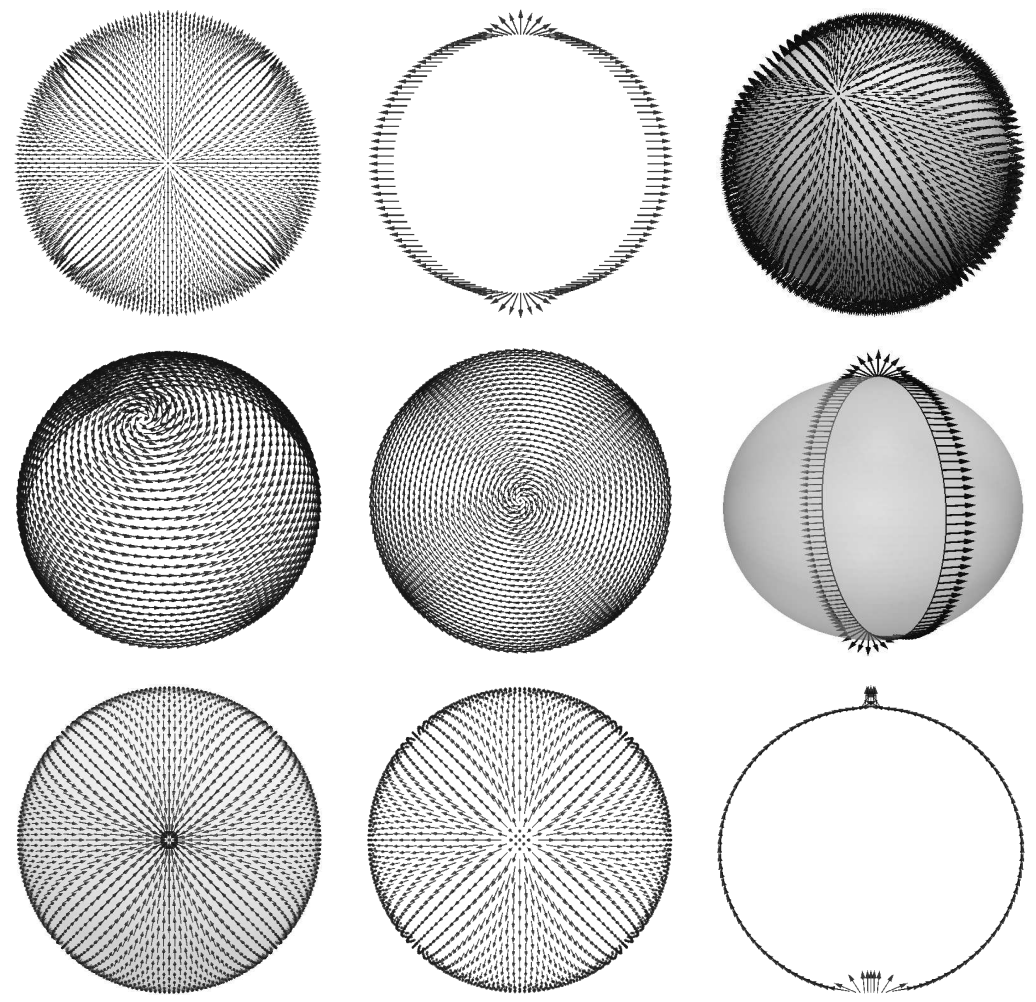

FIG. 10. Initial director fields with two defects of positive degree-one. Upper row: outward pointing defects at north and south pole defined in (i). Middle row: $90^{\circ}$-rotated defects at north and south pole defined in (ii). Lower row: Inward and outward pointing defects at north and south pole defined in (iii). 

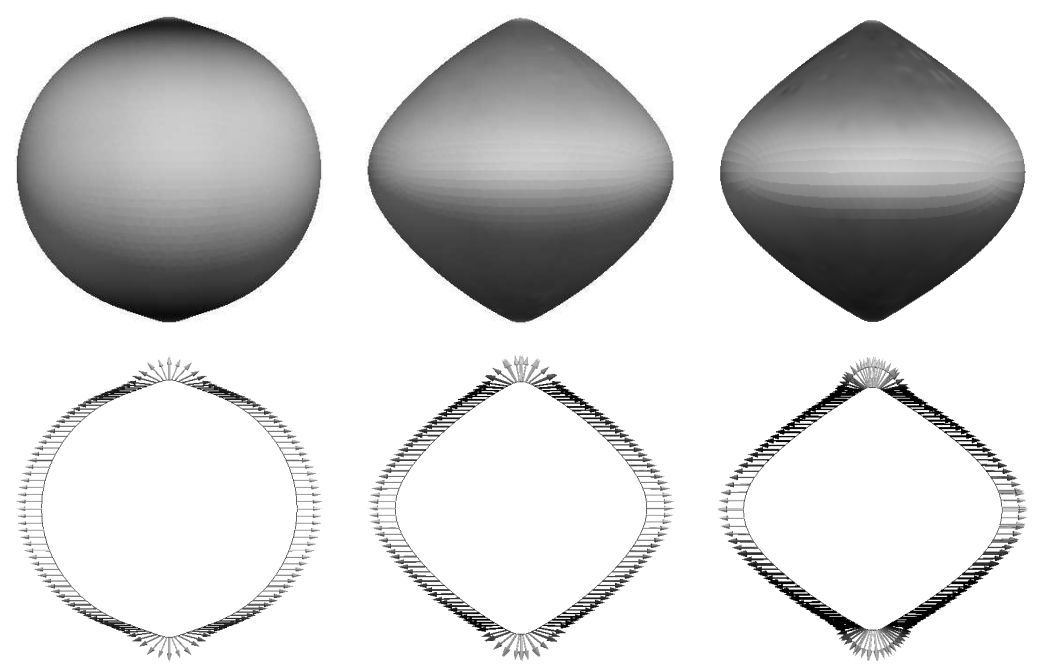

FIG. 11. Biomembrane case with two outward pointing defects of positive degree one: Snapshots of the evolving surface and of the director field along a (deformed) geodesic through the north and south pole after $j=50,1000,4800$ time steps. The surface develops a cone-like shape at the poles while the director field remains nearly unchanged during the evolution. The surface and the director field are colored by $n \cdot v$.
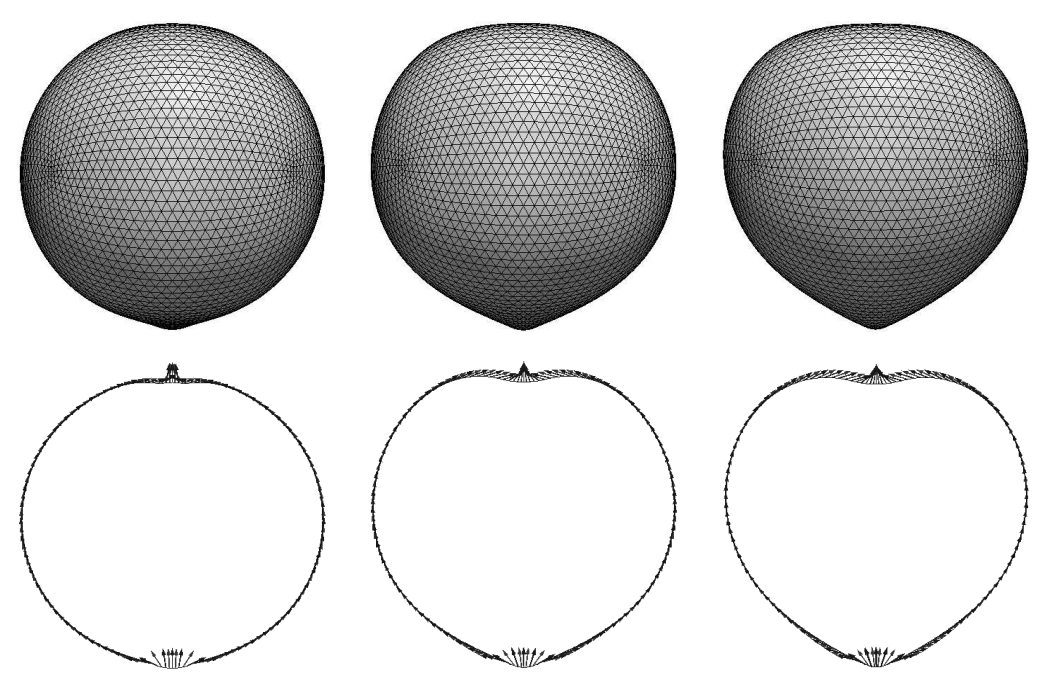

FIG. 12. Biomembrane case with inward and outward pointing defects of positive degree one: Snapshots of the surface and the director field along a (deformed) geodesic through the north and south pole after $j=50,500,1400$ time steps. The surface develops inward and outward cones at the poles while the director field remains nearly unchanged during the evolution.

6.2.2 Negative degree-one defects. To analyze the effect of negative degree-one defects on the local curvature we set $\delta=1$, let $(r, \theta)$ denote polar coordinates of the components $\left(a_{1}, a_{2}\right)$ for a 

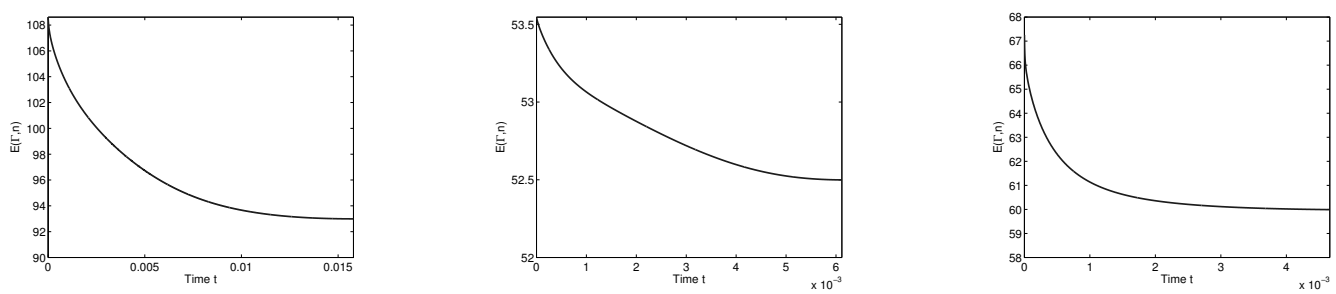

FIG. 13. Decay of energy during the evolution of the positive degree-one defects: Energies for the initial director fields defined through (i),(ii), and (iii) (from left to right).

point $a=\left(a_{1}, a_{2}, a_{3}\right)$ on the sphere, and define the initial director field by setting

$$
\widetilde{n}^{0}(a)=\left(\varphi_{\varepsilon}(r) \cos (-\theta), \varphi_{\varepsilon}(r) \sin (-\theta), \operatorname{sign}\left(a_{3}\right)\left(1-\varphi_{\varepsilon}(r)^{2}\right)^{1 / 2}\right)
$$

and subtracting $90 \%$ of the normal component of $\widetilde{n}^{0}$ so that the resulting director field is nearly tangential, i.e., we set

$$
n^{0}(a)=\frac{\left.\widetilde{n}^{0}(a)-0.9 \widetilde{n}^{0}(a) \cdot a\right) a}{\left.\mid \widetilde{n}^{0}(a)-0.9 \widetilde{n}^{0}(a) \cdot a\right) a \mid} .
$$

The initial director field is constructed in such a way that it has two defects of negative degree one at the poles. It is displayed in Figure 14 from two different perspectives and along the equator. By the Poincaré-Hopf index formula the sum of the degrees of the defects equals the Euler characteristic $\chi$ of the surface. We have $\chi\left(\mathbb{S}^{2}\right)=2$ and for our choice of $n^{0}$ we obtain four defects of positive degree one located on the equator. Two of them are outward and two of them inward pointing. The initial director field is almost stationary for our scheme and the surface adjusts to it during the evolution. From the final configuration displayed in the plots of Figure 15 we see that the surface forms saddles at the north and south pole and cones of positive and negative curvature at the positions of the positive degree-one defects on the equator. This is again in agreement with our predictions for the simplified setting discussed in Section 2.

Acknowledgments. S.B. and A.R. acknowledge support by the DFG through the Collaborative Research Center (SFB) 611 Singular Phenomena and Scaling in Mathematical Models. R.H.N. acknowledges the NSF grant DMS-0807811 and hospitality of the Hausdorff Center for Mathematics. 

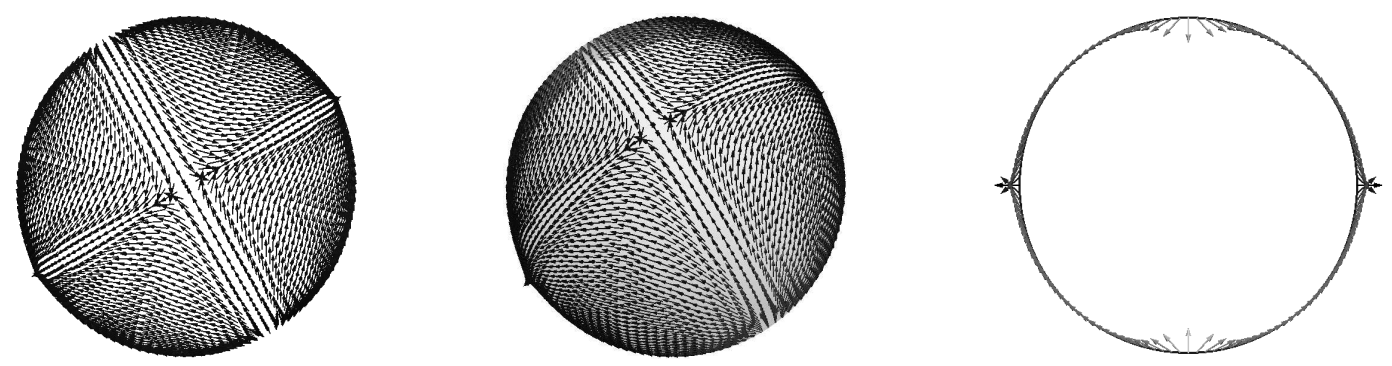

FIG. 14. Initial director field with negative degree-one defects at the north and south pole from different perspectives and the director field along the equator with 4 defects of positive degree one.
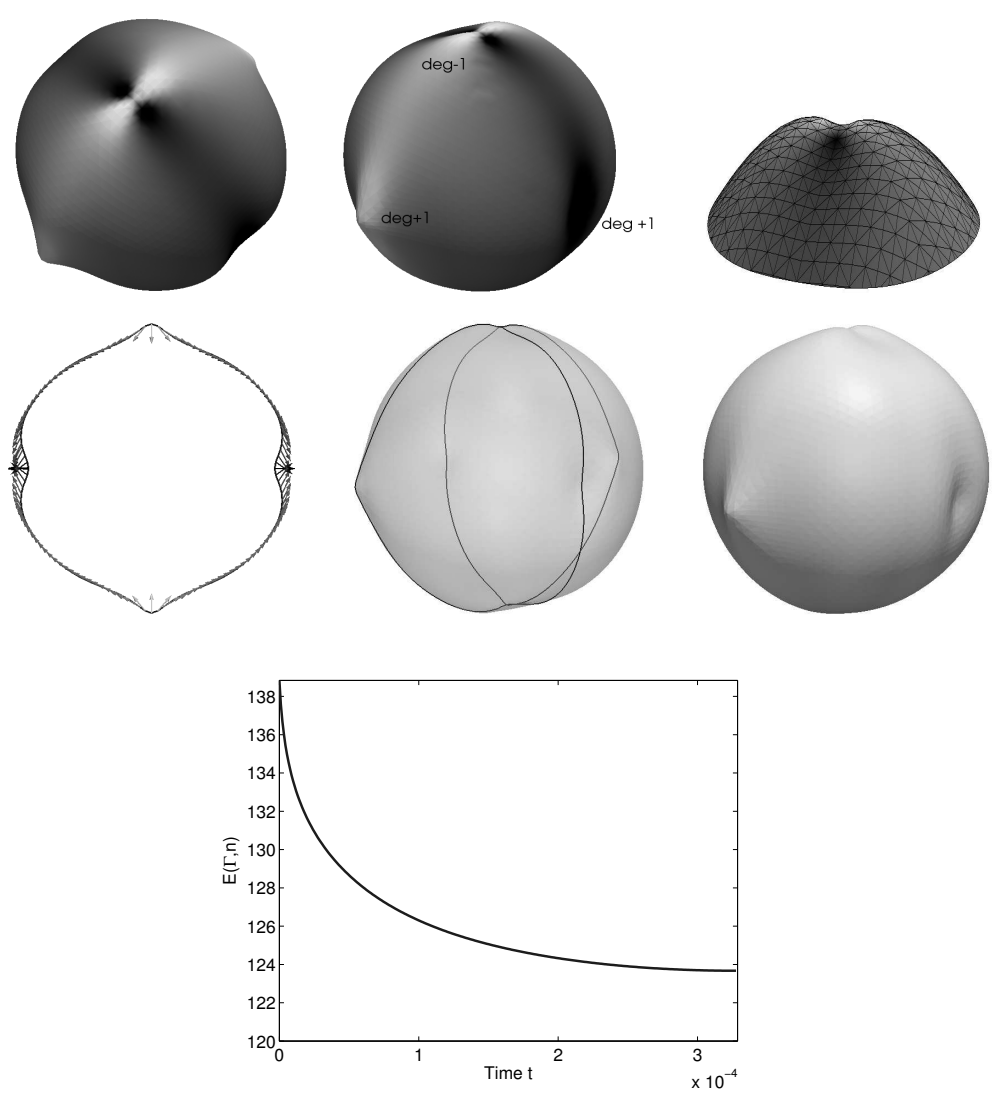

FIG. 15. Final state in the biomembrane case that develops from a director field with two negative degree-one defects at the poles: As in the linearized case the surface forms saddle-shapes at the places where the negative degree-one defects are located (see the zoom to the north-pole in the upper right picture) and cones in neighborhoods of the four positive degree-one defects located on the equator (see the cut through the equator in the lower left picture). The right plot shows a monotone decay of the energy during the evolution. The surface and the director field are colored by $\operatorname{div}_{\Gamma} n$. 


\section{REFERENCES}

1. Alouges, F., A new algorithm for computing liquid crystal stable configurations: the harmonic mapping case. SIAM J. Numer. Anal. 34 (1997), 1708-1726. Zb10886. 35010 MR1472192

2. B̈̈nsch, E., Morin, P. \& Nochetto, R. H., A finite element method for surface diffusion: the parametric case. J. Comput. Phys. 2031 (2005), 321-343. Zbl1070.65093 MR2104399

3. BARrett, J. W., GARCKe, H. \& NÜRnBerg, R., On the parametric finite element approximation of evolving hypersurfaces in $\mathbb{R}^{3}$. J. Comput. Phys. 2279 (2008), 4281-4307. Zb11145. 65068 MR2406538

4. Barrett, J. W., Garcke, H. \& Nürnberg, R., Parametric approximation of Willmore flow and related geometric evolution equations. SIAM J. Sci. Comput. 31 (2008), 225-253. Zbl1186.65133 MR2460777

5. BARtels, S., Stability and convergence of finite-element approximation schemes for harmonic maps. SIAM J. Numer. Anal. 431 (2005), 220-238. Zbl1090.35014 MR2177142

6. Bartels, S., Dolzmann, G. \& Nochetto, R. H., A finite element scheme for the evolution of orientational order in fluid membranes. ESAIM, Math. Model. Numer. Anal. 44 (2010), 1-31. Zblpre05693764 MR2647752

7. Baumgart, T., Hess, S. T. \& WebB, W. W., Imaging co-existing domains in biomembrane models coupling curvature and tension. Nature 425 (2003), 832-824.

8. Bethuel, F., Brezis, H. \& HÉlein, F., Ginzburg-Landau vortices. Progress in Nonlinear Differential Equations and their Applications, 13. Birkhäuser Boston Inc., Boston, MA (1994). Zb10802.35142 MR1269538

9. Bonito, A., Nochetto, R. H. \& Pauletti, M. S., Parametric FEM for geometric biomembranes. Comput. Phys. 229 (2010), 3171-3188. Zblpre05693254 MR2601095

10. Canham, P. B., The minimum energy of bending as a possible explanation of the biconcave shape of the human red blood cell. J. Theort. Biol. 26 (1970), 61-81.

11. Ciarlet, P. G., The finite element method for elliptic problems, vol. 40 of Classics in Applied Mathematics. Society for Industrial and Applied Mathematics (SIAM), Philadelphia, PA (2002). Reprint of the 1978 original [North-Holland, Amsterdam; Zbl0999.65129 MR1930132

12. DoĞAn, G. \& Nochetto, R. H., First variation of the general curvature-dependent surface energy. ESAIM Math. Model. Numer. Anal. 46 (2012), 59-79. MR2846367

13. Du, Q., LiU, C. \& WANG, X., A phase field approach in the numerical study of the elastic bending energy for vesicle membranes. J. Comput. Phys. 198 (2004), 450-468. Zbl1116.74384 MR2062909

14. DzIUK, G., An algorithm for evolutionary surfaces. Numer. Math. 58 (1991), 603-611. Zb10714. 65092 MR1083523

15. DzIUK, G., Computational parametric Willmore flow. Numer. Math. 111 (2008), 55-80. Zb11158. 65073 MR2448203

16. Dziuk, G. \& Elliott, C. M., Finite elements on evolving surfaces. IMA J. Numer. Anal. 27 (2007), 262-292. Zbl1120.65102 MR2317005

17. Elliott, C. M. \& Stinner, B., Modeling and computation of two phase geometric biomembranes using surface finite elements. J. Comput. Phys. 229 (2010), 6585-6612. Zblpre05784817 MR2660322

18. Elliott, C. M. \& Stinner, B., A surface phase field model for two-phase biological membranes. SIAM J. Appl. Math. 70 (2010), 2904-2928. Zbl1209.92003 MR2735109

19. Evans, E., Bending resistance and chemically induced moments in membrane bilayers. Biophys. J. 14 (1974), 923-931.

20. Fournier, J. B. \& GalatoA, P., Sponges, tubules and modulated phases of para-antinematic membranes. J. Phys. II 7 (1997), 1509-1520.

21. Helfrich, W., Elastic properties of lipid bilayers: theory and possible experiments. Z. Naturforsch. C 28 (1973), 693-703. 
22. JEnkins, J. T., The equations of mechanical equilibrium of a model membrane. SIAM J. Appl. Math. 32 (1977), 755-764. Zbl0358.73074 MR0441076

23. Johnson, M. A. \& DeCCA, R. S., Dynamics of topological defects in the $l_{\beta^{\prime}}$ phase of 1, 2-dipalmitoyl phosphatidylcholine bilayers. Optics Communications 281 (2008), 1870-1875.

24. KhAiry, K., Foo, J. \& HowARD, J., Shapes of red blood cells: Comparison of 3 d confocal images with the bilayer-couple model. Cellular and Molecular Bioengineering 1 (2008), 173-181.

25. Ladyzhenskaya, O. A. \& URAL'TSEVA, N. N., Linear and quasilinear elliptic equations. Translated from the Russian by Scripta Technica, Inc. Translation editor: Leon Ehrenpreis. Academic Press, New York, (1968). Zbl0164.13002 MR0244627

26. Laradi, M. \& Mouritsen, O. G., Elastic properties of surfactant monolayers at liquid-liquid interfaces: A molecular dynamics study. J. Chem. Phys. 112 (2000), 8621-8630.

27. Lipowsky, R., DöBereiner, H.-G., Hiergeist, C. \& Indrani, V., Membrane curvature induced by polymers and colloids. Physica A 249 (1998), 536-543.

28. Lowengrub, J. S., RÄtZ, A. \& Voigt, A., Phase-field modeling of the dynamics of multicomponent vesicles: spinodal decomposition, coarsening, budding, and fission. Phys. Rev. E (3) 79 (2009), 0311926. MR2497179

29. Nagle, S. T.-N. J. F., Structure of lipid bilayers. Biochim. Biophys. Acta 1469 (2000), 159-195.

30. Seifert, U., Configurations of fluid membranes and vesicles. Adv. Phys. 46 (1997), 13-137.

31. Struwe, M., Geometric evolution problems. In Nonlinear partial differential equations in differential geometry (Park City, UT, 1992), vol. 2 of IAS/Park City Math. Ser. Amer. Math. Soc., Providence, RI (1996), 257-339. Zb10847.58012 MR1369591

32. UCHIDA, N., Dynamics of orientational ordering in fluid membranes. Phys. Rev. E 66 (Oct 2002), 040902.

33. WANG, X. \& DU, Q., Modelling and simulations of multi-component lipid membranes and open membranes via diffuse interface approaches. J. Math. Biol. 56 (2008), 347-371. Zbl1143.92001 MR2358438

34. Willmore, T. J., An introduction to differential geometry. Clarendon Press, Oxford (1959) reprinted from corrected sheets of the first edition (1959). Zbl0086.14401 MR0159265 